\title{
Convergence to Diffusion Waves of Solutions to Nonlinear Viscoelastic Model with Fading Memory
}

\section{Yanni Zeng}

Courant Institute of Mathematical Sciences, 251 Mercer Street, New York, NY 10012, USA

Received September 12, 1991

\begin{abstract}
We study the large time behavior in $L^{2}$ of solutions to a model for the motion of an unbounded, homogeneous, viscoelastic bar with fading memory. Decay rates for the solutions are obtained under the assumption that the initial data and histories are smooth and small. Moreover, convergence of the solutions to diffusion waves, which are solutions of Burgers equations, is proved and rates are obtained. Our method is based on the study of properties of the solutions to the linearized system in the Fourier space.
\end{abstract}

\section{Introduction}

Consider the following model for the motion of an unbounded, homogeneous, viscoelastic bar with fading memory:

$$
\left\{\begin{array}{l}
u_{t}-v_{x}=0 \\
v_{t}-\sigma_{x}=0, \quad x \in \mathscr{R}, \quad t>0,
\end{array}\right.
$$

where $u, v$, and $\sigma$ are the strain deformation, velocity, and stress; the stress $\sigma$ is a given function of the strain $u$ and its past history,

$$
\sigma(x, t)=f(u(x, t))+\int_{-\infty}^{t} a^{\prime}(t-\tau) g(u(x, \tau)) d \tau ;
$$

here $a(s)$ is a given kernel on $0 \leqq s<\infty$ with derivative $a^{\prime}$, and $f(u), g(u)$ are given smooth material functions. The history and initial data are given by

$$
u(x, t)=\eta(x, t), \quad t \leqq 0, \quad v(x, 0)=v_{0}(x) .
$$

We are interested in the large-time behavior of solutions whose initial data and histories are smooth and specifically, in the convergence of small perturbations (in the integral sense) to a constant state $\bar{U}=(\bar{u}, \bar{v})$ as $t \rightarrow+\infty$, and particularly in 
determining decay rates. Although asymptotic expansions have been proposed to derive a viscous approximation by the hyperbolic-parabolic system

$$
\left\{\begin{array}{l}
u_{t}-v_{x}=0 \\
v_{t}-p(u)_{x}=\left(\mu(u) v_{x}\right)_{x},
\end{array}\right.
$$

where

$$
\begin{aligned}
& p(u)=f(u)-a(0) g(u), \\
& \mu(u)=g^{\prime}(u) \int_{-\infty}^{0} s a^{\prime}(-s) d s,
\end{aligned}
$$

cf. T.-P. Liu [1], there has been no justification of the validity of such an approximation.

In this paper we show that the solution of (1.1), (1.2) has the same large-time behavior as the solution of (1.3), (1.4) with initial data

$$
u(x, 0)=\eta(x, 0), \quad v(x, 0)=v_{0}(x) .
$$

Under appropriate restrictions on $f, g$ and $a$, we show that the solution $U=(u, v)$ of (1.1), (1.2) decays to $\bar{U}$ in $L^{2}$ at rate $(1+t)^{-1 / 4}$, and in $L^{\infty}$ at rate $(1+t)^{-1 / 2}$; moreover, $U$ is asymptotically approximated in $L^{2}$ at rate $(1+t)^{-1 / 2}$ by a combination of $\bar{U}$ and of diffusion waves, which are solutions of Burgers equations. We also obtain corresponding rates for derivatives of the solution. Here our rates are optimal.

First of all, we need to assume that

$$
g^{\prime}(u)>0, \quad p^{\prime}(u)>0, \quad p^{\prime \prime}(u)<0 .
$$

For the kernel $a$, we assume that

$$
\begin{aligned}
& a, a^{\prime}, a^{\prime \prime} \in L^{1}(0, \infty), \\
& \int_{0}^{\infty} t|a(t)| d t<\infty \\
& L\left(a^{\prime}\right)(z) \neq 0, \quad \forall z \in \Pi, \\
& a \text { is strongly positive definite, }
\end{aligned}
$$

where $L\left(a^{\prime}\right)$ denotes the Laplace transform of $a^{\prime}, \Pi \equiv\{z \in \mathscr{C}: \operatorname{Re} z \geqq 0\}$, and the definition of strong positivity will be given below. These assumptions include the situation of physical interest. Global existence and uniqueness of a classical solution has been established by Hrusa and Nohel [5] under (1.5), (1.6) ${ }_{1}$ and suitable regularity and smallness assumptions on the initial data and past history. They showed the decay of $U$ to $\bar{U}$ in $L^{\infty}$. However, they don't provide decay rates since their proof was based on energy estimates. To obtain the decay rates here we further assume that

$$
L(\text { a) is a rational fraction; }
$$

it is not clear whether $(1.6)_{2}$ is necessary for obtaining decay rates or not. However, it is satisfied in the most important physical application where $a$ is a finite sum of decaying exponential functions with positive coefficients.

Let's explain the conditions on $a$. First we have 
Definition. A function $a \in L_{\mathrm{loc}}^{1}[0, \infty)$ is said to be positive definite if

$$
\int_{0}^{t} y(s) \int_{0}^{s} a(s-\tau) y(\tau) d \tau d s \geqq 0, \quad \forall t \geqq 0,
$$

for every $y \in C[0, \infty) ; a$ is called strongly positive definite if there exists a constant $c>0$ such that the function defined by $a(t)-c e^{-t}, t \geqq 0$, is positive definite.

The definition is generally not easy to check directly. For our purposes here, it is useful to know the following

Lemma. If $a \in L^{1}(0, \infty)$, then $a$ is strongly positive definite if and only if there exists a constant $c>0$ such that

$$
\operatorname{Re}[L(a)(i \omega)] \geqq \frac{c}{\omega^{2}+1}, \quad \forall \omega \in \mathscr{R}
$$

Using (1.7) and the regularity on $a$ one can check that $(1.6)_{1}$ implies

$$
a(0)>0, \quad a^{\prime}(0)<0 .
$$

See [5] and the references therein; (1.6) implies that $a$ decays exponentially, i.e., there is a constant $C>0$ such that

$$
|a(t)| \leqq C e^{-t / C}, \quad \forall t \geqq 0 .
$$

This can be seen from the inverse Laplace transform

$$
a(t)=\frac{1}{2 \pi i} \int_{-i \infty}^{i \infty} L(a)(s) e^{s t} d s
$$

(1.6) and the residue theorem. Similarly, we have

$$
\left|a^{\prime}(t)\right|,\left|a^{\prime \prime}(t)\right| \leqq C e^{-t / C}, \quad \forall t \geqq 0 .
$$

Conditions $(1.6)_{1}$ and $(1.6)_{2}$ are satisfied by kernels of the form

$$
a(t)=\sum_{j=1}^{N} \alpha_{j} e^{-\mu_{j} t}, \quad t \geqq 0,
$$

with $\alpha_{j}, \mu_{j}>0, j=1, \ldots, N$, which are commonly used in applications of viscoelasticity theory. Moreover, they are also satisfied by oscillatory kernels of the form $e^{-\mu t} \cos \beta t, \mu>0$.

Set

$$
\begin{gathered}
U_{0}(x)=\left(u_{0}(x), v_{0}(x)\right), \quad u_{0}(x) \equiv \eta(x, 0), \\
E_{s}=\left\|U_{0}-\bar{U}\right\|_{L^{1}}+\left\|v_{0}-\bar{v}\right\|_{s}+\sup _{\tau \leqq 0}\|\eta-\bar{u}\|_{s}(\tau),
\end{gathered}
$$

where $\|\cdot\|_{L^{p}}$ denotes the $L^{p}$-norm on $\mathscr{R},\|\cdot\| \equiv\|\cdot\|_{L^{2}}$, and $\|\cdot\|_{s}$ is the Sobolev norm:

$$
\|h\|_{s}=\left\{\sum_{j=0}^{s}\left\|D^{j} h\right\|^{2}\right\}^{1 / 2}, \quad D^{l} \equiv \partial^{l} / \partial x^{l} .
$$

Our first result is about the $L^{2}$ decay of the solution. 
Theorem 1.1. Assume that (1.5) and (1.6) hold, $\eta$ and $v_{0}$ satisfy

$$
\begin{gathered}
v_{0}-\bar{v} \in H^{s}(\mathscr{R}), \quad U_{0}-\bar{U} \in L^{1}(\mathscr{R}), \\
\eta-\bar{u} \in L^{\infty}\left((-\infty, 0] ; H^{s}(\mathscr{R})\right) \cap C\left((-\infty, 0] ; H^{s}(\mathscr{R})\right),
\end{gathered}
$$

where $s \geqq 3$. If $E_{s}$ is sufficiently small, then (1.1), (1.2) has a unique solution $U$ defined on $\mathscr{R} \times[0, \infty)$, with $U-\bar{U} \in C\left([0, \infty) ; H^{s}(\mathscr{R})\right) \cap C^{1}\left([0, \infty) ; H^{s-1}(\mathscr{R})\right), U_{x} \in L^{2}([0, \infty)$; $\left.H^{s-1}(\mathscr{R})\right)$, and

$$
\left\|D^{k}(U-\bar{U})\right\|_{s-2-2 k}(t) \leqq C E_{s}(1+t)^{-1 / 2(k+1 / 2)}, \quad t \geqq 0,
$$

where $0 \leqq k \leqq s / 2-1$ and $C$ is a constant.

Clearly, the $L^{\infty}$ decay rate $(1+t)^{-1 / 2}$ of $U-\bar{U}$ is a consequence of the estimate (1.12) and the Sobolev inequality.

Before stating our second result, let's introduce the parabolic system

$$
\left\{\begin{array}{l}
\tilde{u}_{t}-\tilde{v}_{x}=\frac{\mu}{2} \tilde{u}_{x x} \\
\tilde{v}_{t}-p(\tilde{u})_{x}=\frac{\mu}{2} \tilde{v}_{x x}
\end{array}\right.
$$

and the hyperbolic system

$$
\left\{\begin{array}{l}
u_{t}-v_{x}=0 \\
v_{t}-p(u)_{x}=0
\end{array}\right.
$$

corresponding to (1.1), where $\mu \equiv \mu(\bar{u}), \mu(u)$ and $p(u)$ are defined by (1.4). By (1.5) and (1.7),

$$
\mu=g^{\prime}(\bar{u}) \int_{0}^{\infty} a(t) d t=g^{\prime}(\bar{u}) L(a)(0)>0 .
$$

We are going to discuss the large-time behavior of the solution to (1.13) with initial data

$$
\tilde{U}(x, 0)=U_{0}(x)
$$

where $\tilde{U}=(\tilde{u}, \tilde{v})$, and show that it is the same as the large-time behavior of the solution to (1.1), (1.2). We choose (1.13) instead of (1.3) for technical reasons. They have the same asymptotic behavior in $L^{2}$ if they have the same initial data (cf. S. Kawashima [2]); however, (1.13) is more convenient to handle since it is uniformly parabolic.

The hyperbolic system (1.14) has eigenvalues

$$
\lambda_{1,2}=\mp \sqrt{p^{\prime}(u)}
$$

right eigenvectors

$$
r_{1,2}=\frac{2 \sqrt{p^{\prime}(u)}}{p^{\prime \prime}(u)}\left(\begin{array}{c}
\mp 1 \\
-\sqrt{p^{\prime}(u)}
\end{array}\right),
$$


and left eigenvectors

$$
l_{1,2}=-\frac{p^{\prime \prime}(u)}{4 \sqrt{p^{\prime}(u)}}\left( \pm 1, \frac{1}{\sqrt{p^{\prime}(u)}}\right),
$$

satisfying $l_{i} \cdot r_{j}=\delta_{i j}, i, j=1,2$, and $\nabla \lambda_{i} \cdot r_{i}=1, i=1,2$. We define the diffusion waves for (1.13), (1.16) as

$$
\psi_{i}(x, t)=\theta_{i}(x, t) r_{i}(\bar{u}), \quad i=1,2,
$$

where $r_{i}$ are the right eigenvectors of (1.14), and $\theta_{i}$ is the solution of the initial value problem

$$
\begin{gathered}
\frac{\partial \theta_{i}}{\partial t}+\lambda_{i}(\bar{u}) \frac{\partial \theta_{i}}{\partial x}+\frac{\partial}{\partial x}\left(\frac{\theta_{i}^{2}}{2}\right)=\frac{\mu}{2} \frac{\partial^{2} \theta_{i}}{\partial x^{2}}, \\
\theta_{i}(x, 0)=l_{i}(\bar{u})\left(U_{0}(x)-\bar{U}\right) .
\end{gathered}
$$

Set

$$
\Psi(x, t)=\bar{U}+\psi_{1}(x, t)+\psi_{2}(x, t) .
$$

Our second result concerns the approximation of $U$ by $\Psi$.

Theorem 1.2. Assume that the hypotheses of Theorem 1.1 are in force with $s \geqq 4$ and $x\left(U_{0}-\bar{U}\right) \in L^{1}(\mathscr{R})$. Set

$$
E_{s}^{*}=E_{s}+\int_{-\infty}^{\infty}\left|x\left(U_{0}(x)-\bar{U}\right)\right| d x .
$$

If $E_{s}^{*}$ is sufficiently small, then the solution $U$ for (1.1), (1.2) satisfies

$$
\left\|D^{k}(U-\Psi)\right\|_{s-4-2 k}(t) \leqq C E_{s}^{*}(1+t)^{-1 / 2(k+1)}
$$

for $t \geqq 0$, where $0 \leqq k \leqq s / 2-2$, and $C$ is a constant.

Theorem 1.2 gives us a complete picture for the asymptotic behavior of the solution $U$ to the viscoelastic model $(1.1),(1.2)$ in the $L^{2}$ sense. It tells us $U$ converges to $\Psi$ at the same optimal rate as the solution $\tilde{U}$ of the parabolic system (1.13), (1.16) does, cf. Theorem 1.3 below. Our strategy for proving it is to show that $U-\tilde{U}$ decays faster than $\tilde{U}-\Psi$.

Diffusion waves were constructed by T.-P. Liu [6] (Also see [3 and 4]). Asymptotic behavior of solutions to parabolic systems has been studied in $L^{p}, 1 \leqq p \leqq \infty$, by I.-L. Chern and T.-P. Liu [4], T.-P. Liu [3], where optimal rates were obtained in [3]. Asymptotic behavior of solutions to hyperbolic-parabolic systems like (1.3), (1.16) has been studied in $L^{2}$ by Kawashima [2]. However, his rate for the convergence to $\Psi$ is not optimal. For our purpose here we cite some facts from [2, 3 and 4$]$ concerning the solution for (1.13), (1.16) as the following

Theorem 1.3. Consider the initial value problem (1.13), (1.16).

i) Assume $U_{0}-\bar{U} \in H^{s}(\mathscr{R}) \cap L^{1}(\mathscr{R})$ for $s \geqq 1$. If

$$
\tilde{E}_{s}=\left\|U_{0}-\bar{U}\right\|_{s}+\left\|U_{0}-\bar{U}\right\|_{L^{1}}
$$

is small, then (1.13), (1.16) has a unique global solution $\tilde{U}(x, t)$ satisfying $\tilde{U}-\bar{U} \epsilon$ $C\left([0, \infty) ; H^{s}(\mathscr{R})\right)\left(\cap C^{1}\left([0, \infty) ; H^{s-2}(\mathscr{R})\right)\right.$ if $\left.s \geqq 2\right)$ and $D \tilde{U} \in L^{2}\left([0, \infty) ; H^{s}(\mathscr{R})\right)$. The 
solution satisfies the decay estimate

$$
\left\|D^{k}(\tilde{U}-\bar{U})\right\|_{s-k} \leqq C \tilde{E}_{s}(1+t)^{-1 / 2(k+(1 / 2))}, \quad t \geqq 0,
$$

where $0 \leqq k \leqq s$ and $C$ is a constant.

ii) Assume that $U_{0}$ has the property that

$$
\delta \equiv \int_{-\infty}^{\infty}\left[\left|U_{0}(x)-\bar{U}\right|+\left|x\left(U_{0}(x)-\bar{U}\right)\right|+\left|U_{0}(x)-\bar{U}\right|^{2}+\left|U_{0}^{\prime}(x)\right|^{2}\right] d x
$$

is small. Then the solution $\tilde{U}$ for $(1.13),(1.16)$ exists with $\tilde{U}-\bar{U} \in C\left((0, \infty) ; H^{k}(\mathscr{R})\right) \cap$ $L^{2}\left((0, \infty) ; H^{k+1}(\mathscr{R})\right)$ and satisfies

$$
\left\|D^{k}(\tilde{U}-\Psi)\right\|_{L^{p}}(t) \leqq C \delta t^{-1 / 2(k+(3 / 2)-(1 / p))}, \quad t>0,
$$

where $1 \leqq p \leqq \infty, C$ is a constant.

We will show that the solution of the viscoelastic model is approximated by the solution of the uniformly parabolic system (1.13), (1.16) at least at the same rate as in (1.21) by proving the following result.

Theorem 1.4. Assume that the hypotheses of Theorem 1.1 are in force with $s \geqq 4$. Moreover, assume that $\eta-\bar{u} \in L^{\infty}\left((-\infty, 0] ; L^{r}(\mathscr{R})\right) \cap C\left((-\infty, 0] ; L^{r}(\mathscr{R})\right)$, where $1<r \leqq 2$. Set

$$
E_{s, r}=E_{s}+\sup _{\tau \leqq 0}\|\eta-\bar{u}\|_{L^{r}}(\tau) .
$$

If $E_{s, r}$ is sufficiently small, then the solution $U$ for (1.1), (1.2) and the solution $\tilde{U}$ for (1.13), (1.16) satisfy

$$
\left\|D^{k}(U-\tilde{U})\right\|_{s-4-2 k}(t) \leqq C E_{s, r}(1+t)^{-1 / 2(k+(1 / r)+(1 / 2))},
$$

where $0 \leqq k \leqq s / 2-2, C$ is a constant.

Remark. Theorem 1.2 is a consequence of Theorem 1.4 with $r=2$, Theorem 1.3, and Theorem 1.1.

Remark. If $\eta-\bar{u} \in L^{\infty}\left((-\infty, 0] ; L^{1}(\mathscr{R})\right) \cap C\left((-\infty, 0] ; L^{1}(\mathscr{R})\right)$ and $E_{s, 1}$ is sufficiently small, then we obtain the decay rate $(1+t)^{-1 / 2(k+(3 / 2))+\alpha}$ in Theorem 1.4 , with $\alpha>0$ arbitrarily small. This is the same decay rate as hyperbolic-parabolic systems are approximated by uniformly parabolic systems. See [2] Theorem 6.3.

To prove Theorems 1.1 and 1.4, the key step is to obtain appropriate decay estimates for the linearized systems (2.1)-(2.3) and (2.41), (2.42) below; these are linearizations of systems (1.1) and (1.13) respectively about the constant state $\bar{U}$. This is a serious difficulty for the viscoelastic model problem since an explicit formula for solutions of the linearized systems is not available, even for quite special kernels like a sum of exponential functions; by contrast, in the case of parabolic and hyperbolic-parabolic systems of PDE's, explicit formulas are available and estimates for the linearized systems are straightforward.

The plan of this paper is as follows: In Sect. 2, we discuss solutions of relevant linearized systems in detail. In Sect. 3, we generalize the energy estimate of Hrusa and Nohel [5] to higher derivatives (Also see Dafermos and Nohel [8].) Finally in Sect. 4, we prove Theorems 1.1 and 1.4.

For the general theory of (1.1) the reader is referred to the book of Renardy, Hrusa, and Nohel [7]. 


\section{Solutions for Linear Equations}

To obtain the decay estimate for the system (1.1), we are going to linearize it about the constant state $\bar{U}$, and to prove that the solution for (1.1) has the same decay rate as the solution for the linearization. Therefore it is crucial to obtain the decay estimate for the linearization, which is our purpose in this section.

After linearizing (1.1), we consider the following initial value problem:

$$
\begin{aligned}
& \left\{\begin{array}{l}
u_{t}-v_{x}=0 \\
v_{t}-f^{\prime} u_{x}=g^{\prime} \int_{0}^{t} a^{\prime}(t-\tau) u_{x}(x, \tau) d \tau+\phi, \quad x \in \mathscr{R}, \quad t>0,
\end{array}\right. \\
& U(x, 0)=U_{0}(x), \quad x \in \mathscr{R},
\end{aligned}
$$

where $U \equiv(u, v), U_{0} \equiv\left(u_{0}, v_{0}\right), f^{\prime}=f^{\prime}(\bar{u})$ and $g^{\prime}=g^{\prime}(\bar{u})$ are constants satisfying

$$
g^{\prime}>0, \quad p^{\prime} \equiv f^{\prime}-a(0) g^{\prime}>0,
$$

$a$ is a given kernel satisfying.(1.6), $\phi$ is a given smooth function of $x$ and $t$, and $U_{0}(x)$ is a small perturbation of zero in the integral sense.

In this section, Fourier transform and Laplace transform are used to explore properties of the solution to (2.1), (2.2). Let's assume $U_{0} \in L^{1}(\mathscr{R}) \cap H^{s}(\mathscr{R})$ and $\phi \in C\left([0, \infty) ; H^{s}(\mathscr{R})\right), s \geqq 2$. First we take Fourier transform with respect to $x$ denoted by " $\wedge$ " to (2.1), (2.2):

$$
\begin{aligned}
& \left\{\begin{array}{l}
u_{t}^{\wedge}-i \xi v^{\wedge}=0 \\
v_{t}^{\wedge}-f^{\prime} i \xi u^{\wedge}=g^{\prime} \int_{0}^{t} a^{\prime}(t-\tau) i \xi u^{\wedge}(\xi, \tau) d \tau+\phi^{\wedge},
\end{array}\right. \\
& U^{\wedge}(\xi, 0)=U_{\hat{0}}^{\wedge}(\xi) .
\end{aligned}
$$

Then take Laplace transform formally to (2.4),

$$
\begin{aligned}
s L\left(u^{\wedge}\right)-u_{0}^{\wedge}-i \xi L\left(v^{\wedge}\right) & =0, \\
s L\left(v^{\wedge}\right)-v_{0}^{\wedge}-f^{\prime} i \xi L\left(u^{\wedge}\right) & =g^{\prime} i \xi L\left(a^{\prime}\right) L\left(u^{\wedge}\right)+L\left(\phi^{\wedge}\right) .
\end{aligned}
$$

This is an algebraic system. It can be solved easily to get

$$
\begin{aligned}
L\left(u^{\wedge}\right) & =\frac{s u_{0}^{\wedge}+i \xi v_{0}^{\wedge}+i \xi L\left(\phi^{\wedge}\right)}{s^{2}+\xi^{2}\left[g^{\prime} L\left(a^{\prime}\right)+f^{\prime}\right]}, \\
L\left(v^{\wedge}\right) & =\frac{i \xi\left[g^{\prime} L\left(a^{\prime}\right)+f^{\prime}\right] u_{0}^{\wedge}+s v_{0}^{\wedge}+s L\left(\phi^{\wedge}\right)}{s^{2}+\xi^{2}\left[g^{\prime} L\left(a^{\prime}\right)+f^{\prime}\right]} .
\end{aligned}
$$

Taking the inverse transform and using the convolution theorem, we arrive at the following formula,

$$
\left\{\begin{array}{l}
u^{\wedge}(\xi, t)=u_{0}^{\wedge}(\xi) \eta_{1}(\xi, t)+v_{0}^{\wedge}(\xi) \eta_{2}(\xi, t)+\int_{0}^{t} \eta_{2}(\xi, t-\tau) \phi^{\wedge}(\xi, \tau) d \tau \\
v^{\wedge}(\xi, t)=u_{0}^{\wedge}(\xi) \eta_{3}(\xi, t)+v_{0}^{\wedge}(\xi) \eta_{1}(\xi, t)+\int_{0}^{t} \eta_{1}(\xi, t-\tau) \phi^{\wedge}(\xi, \tau) d \tau,
\end{array}\right.
$$


where

$$
\begin{aligned}
& \eta_{1}(\xi, t)=\frac{1}{2 \pi i} \int_{\sigma-i \infty}^{\sigma+i \infty} \frac{s e^{s t}}{s^{2}+g^{\prime} \xi^{2} s L(a)(s)+p^{\prime} \xi^{2}} d s \\
& \eta_{2}(\xi, t)=\frac{i \xi}{2 \pi i} \int_{\sigma-i \infty}^{\sigma+i \infty} \frac{e^{s t}}{s^{2}+g^{\prime} \xi^{2} s L(a)(s)+p^{\prime} \xi^{2}} d s \\
& \eta_{3}(\xi, t)=\frac{i \xi}{2 \pi i} \int_{\sigma-i \infty}^{\sigma+i \infty} \frac{e^{s t}\left[g^{\prime} s L(a)(s)+p^{\prime}\right]}{s^{2}+g^{\prime} \xi^{2} s L(a)(s)+p^{\prime} \xi^{2}} d s
\end{aligned}
$$

$\sigma$ is a real number sufficiently large.

To obtain estimates on $U^{\wedge}$, we need a more specific form of $\eta$ 's. By assumption (1.6), we can write

$$
L(a)=q_{1} / q_{2},
$$

where $q_{1}$ and $q_{2}$ are polynomials that are relatively prime and are of degree $m_{1}$ and $m_{2}$ respectively and the coefficient of the highest power in $q_{2}$ is 1 . Clearly we must have $m_{1}<m_{2}$ since $a \in L^{1}(0, \infty)$; moreover, $q_{1}$ and $q_{2}$ have real coefficients since $a$ is real. Using (2.9),

$$
\eta_{1}(\xi, t)=\frac{1}{2 \pi i} \int_{\sigma-i \infty}^{\sigma+i \infty} \frac{s q_{2}(s) e^{s t}}{d(s ; \xi)} d s
$$

where

$$
d(s ; \xi)=s^{2} q_{2}(s)+g^{\prime} \xi^{2} s q_{1}(s)+p^{\prime} \xi^{2} q_{2}(s)
$$

is a polynomial in $s$ having degree $n=m_{2}+2$.

Lemma 2.1. Assume that (2.3) and (1.6) hold. Denote the $n$ zeros of $d(s ; \xi)$ on s-plane as $\lambda_{k}(\xi), k=1, \ldots, n$. Then $\lambda_{k}(\xi), k=1, \ldots, n$, has the following properties:

i) If $\xi \neq 0, \lambda_{k}(\xi)$ is not a zero of $q_{2}$..

ii) $\lambda_{k}(\xi)$ is a simple zero of $d(s ; \xi)$ except at a finite number of values $\xi$.

Proof. i) If $\lambda_{k}(\xi)$ is a zero of $q_{2}$, substituting it into (2.11) we get $g^{\prime} \xi^{2} \lambda_{k} q_{1}\left(\lambda_{k}\right)=0$. Here $g^{\prime}>0$ by (2.3), $\xi^{2} \neq 0$ by the assumption, and $q_{1}\left(\lambda_{k}\right) \neq 0$ since $q_{1}$ and $q_{2}$ are relatively prime. Therefore $\lambda_{k}=0$. But then $q_{2}\left(\lambda_{k}\right)=0$ is a contradiction to $a \in L^{1}(0, \infty)$.

ii) If $\lambda_{k}(\xi)$ is a double zero, then

$$
\begin{gathered}
d\left(\lambda_{k} ; \xi\right)=\lambda_{k}^{2} q_{2}\left(\lambda_{k}\right)+\xi^{2}\left[g^{\prime} \lambda_{k} q_{1}\left(\lambda_{k}\right)+p^{\prime} q_{2}\left(\lambda_{k}\right)\right]=0 \\
d^{\prime}\left(\lambda_{k} ; \xi\right)=2 \lambda_{k} q_{2}\left(\lambda_{k}\right)+\lambda_{k}^{2} q_{2}^{\prime}\left(\lambda_{k}\right)+\xi^{2}\left[g^{\prime} q_{1}\left(\lambda_{k}\right)+g^{\prime} \lambda_{k} q_{1}^{\prime}\left(\lambda_{k}\right)+p^{\prime} q_{2}^{\prime}\left(\lambda_{k}\right)\right]=0
\end{gathered}
$$

where "'" denotes the derivative with respect to $s$.

The quantity in the bracket in (2.12) can't be zero. In fact, if it is zero and $\xi \neq 0$, then (2.12) gives us $\lambda_{k}^{2} q_{2}\left(\lambda_{k}\right)=0$. By i) $q_{2}\left(\lambda_{k}\right) \neq 0$. Therefore $\lambda_{k}=0$. Substituting back to the bracket we get $p^{\prime} q_{2}\left(\lambda_{k}\right)=0$. It is impossible since $p^{\prime}>0$. If $\xi=0$, either $\lambda_{k}(0)=0$ or $q_{2}\left(\lambda_{k}\right)=0$ by $(2.11)$. Clearly the bracket in (2.12) can't be zero. We solve for $\xi^{2}$ in terms of $\lambda_{k}$ from (2.12),

$$
\xi^{2}=\frac{-\lambda_{k}^{2} q_{2}\left(\lambda_{k}\right)}{g^{\prime} \lambda_{k} q_{1}\left(\lambda_{k}\right)+p^{\prime} q_{2}\left(\lambda_{k}\right)} .
$$


The bracket in (2.13) is a polynomial of $\lambda_{k}$. It is not zero except of a finite number of $\lambda_{k}$, or by (2.14), except of a finite number of $\xi$. Therefore

$$
\xi^{2}=\frac{-2 \lambda_{k} q_{2}\left(\lambda_{k}\right)-\lambda_{k}^{2} q_{2}^{\prime}\left(\lambda_{k}\right)}{g^{\prime} q_{1}\left(\lambda_{k}\right)+g^{\prime} \lambda_{k} q_{1}^{\prime}\left(\lambda_{k}\right)+p^{\prime} q_{2}^{\prime}\left(\lambda_{k}\right)}
$$

except of a finite number of $\xi$.

Set the right-hand sides of (2.14) and (2.15) equal. $\lambda_{k}$ can take at most a finite number of values. So does $\xi$. Q.E.D

Fix $\xi$ and take $\sigma>\operatorname{Re} \lambda_{k}(\xi), k=1, \ldots, n$ in (2.10). Then use residue theorem and note that $\lambda_{k}(\xi), k=1, \ldots, n$, are simple poles except at a finite number of $\xi$ 's. We arrive at

$$
\eta_{1}(\xi, t)=\sum_{k=1}^{n} \frac{\lambda_{k} q_{2}\left(\lambda_{k}\right) e^{\lambda_{k} t}}{d^{\prime}\left(\lambda_{k} ; \xi\right)}
$$

except of a finite number of $\xi$ 's. We have similar expressions for $\eta_{2}$ and $\eta_{3}$ in (2.8). Substituting them in (2.7) we have

Lemma 2.2. Assume that the hypotheses of Lemma 2.1 are in force. Then except at a finite number of $\xi$ 's, the solution for (2.4), (2.5) is

$$
\begin{aligned}
u^{\wedge}(\xi, t)= & u_{0}^{\wedge}(\xi) \sum_{k=1}^{n} \frac{\lambda_{k} q_{2}\left(\lambda_{k}\right)}{d^{\prime}\left(\lambda_{k} ; \xi\right)} e^{\lambda_{k} t}+v_{0}^{\wedge}(\xi) \sum_{k=1}^{n} \frac{i \xi q_{2}\left(\lambda_{k}\right)}{d^{\prime}\left(\lambda_{k} ; \xi\right)} e^{\lambda_{k} t} \\
& +\int_{0}^{t} \sum_{k=1}^{n} \frac{i \xi q_{2}\left(\lambda_{k}\right)}{d^{\prime}\left(\lambda_{k} ; \xi\right)} e^{\lambda_{k}(t-\tau)} \phi^{\wedge}(\xi, \tau) d \tau \\
v^{\wedge}(\xi, t)= & u_{0}^{\wedge}(\xi) \sum_{k=1}^{n} \frac{i \xi\left[g^{\prime} \lambda_{k} q_{1}\left(\lambda_{k}\right)+p^{\prime} q_{2}\left(\lambda_{k}\right)\right]}{d^{\prime}\left(\lambda_{k} ; \xi\right)} e^{\lambda_{k} t}+v_{0}^{\wedge}(\xi) \sum_{k=1}^{n} \frac{\lambda_{k} q_{2}\left(\lambda_{k}\right)}{d^{\prime}\left(\lambda_{k} ; \xi\right)} e^{\lambda_{k} t} \\
& +\int_{0}^{t} \sum_{k=1}^{n} \frac{\lambda_{k} q_{2}\left(\lambda_{k}\right)}{d^{\prime}\left(\lambda_{k} ; \xi\right)} e^{\lambda_{k}(t-\tau)} \phi^{\wedge}(\xi, \tau) d \tau,
\end{aligned}
$$

where $q_{1}$ and $q_{2}$ are defined by (2.9), $d(s ; \xi)$ by $(2.11)$, and $\lambda_{k}=\lambda_{k}(\xi), k=1, \ldots, n$, are zeros of $d$.

Remark. We have derived (2.16) by taking Laplace transform formally to (2.4). Actually at that point we didn't know if the transform exists or not. However, once we get (2.16), it can be checked by direct substitution that it is a solution to (2.4), (2.5). Therefore the inverse Fourier transform of $(2.16)$ gives us a $C\left([0, \infty) ; H^{s}(\mathscr{R})\right) \cap$ $C^{1}\left([0, \infty) ; H^{s-1}(\mathscr{R})\right)$ solution to $(2.1),(2.2)$, which is unique, cf. [5] or Theorem 3.2 below.

Set

$$
\mu \equiv g^{\prime} \int_{0}^{\infty} a(t) d t
$$

Then $\mu=g^{\prime} L(a)(0)>0$ by (2.3) and (1.7).

Lemma 2.3. Assume that the hypotheses of Lemma 2.1 are in force and $\xi$ is real. Then we have the following properties: 
i) For small $\delta>0$,

$$
\lambda_{1,2}(\xi)=\mp i \sqrt{p^{\prime}} \xi-\frac{1}{2} \mu \xi^{2}+O\left(\xi^{3}\right), \quad|\xi| \leqq \delta,
$$

and there exists a constant $\alpha=\alpha(\delta)>0$, such that

$$
\begin{gathered}
\operatorname{Re} \lambda_{1,2}(\xi) \leqq-\alpha, \quad|\xi| \geqq \delta, \\
\operatorname{Re} \lambda_{k}(\xi) \leqq-\alpha, \quad k=3, \ldots, n, \quad \xi \in \mathscr{R} .
\end{gathered}
$$

ii) $\lambda_{1}(\xi)$ and $\lambda_{2}(\xi)$ are complex conjugates with

$$
\lim _{\xi \rightarrow \infty}\left(\lambda_{1,2}(\xi) / \xi\right)^{2}=-f^{\prime}
$$

while the limits of the other zeros exist,

$$
\lim _{\xi \rightarrow \infty} \lambda_{k}(\xi)=\lambda_{k}^{0}, \quad k=3, \ldots, n,
$$

where $\lambda_{k}^{0}, k=3, \ldots, n$, are the $m_{2}=n-2$ zeros of

$$
q(s) \equiv p^{\prime} q_{2}(s)+g^{\prime} s q_{1}(s) .
$$

Proof. i) First $\lambda_{k}(\xi), k=1, \ldots, n$, are continuous functions of $\xi$ since the coefficients in $d(s ; \xi)$ are continuous on $\xi$. Note that

$$
d(s ; 0)=s^{2} q_{2}(s) \text {. }
$$

Therefore $\lambda_{1}(0)=\lambda_{2}(0)=0$, while $\lambda_{k}(0), k=3, \ldots, n$, are zeros of $q_{2}$. Since $a \in L^{1}(0, \infty)$,

$$
\operatorname{Re} \lambda_{k}(0)<0, \quad k=3, \ldots, n \text {. }
$$

Regard $\xi$ as a complex variable for a moment. Then in a small neighborhood of zero we have

$$
\xi=\lambda_{1} h_{1}\left(\lambda_{1}\right), \quad \xi=\lambda_{2} h_{2}\left(\lambda_{2}\right)
$$

via (2.14), where $h_{1}$ and $h_{2}$ are analytic functions with

$$
h_{1}(0)=\frac{i}{\sqrt{p^{\prime}}}, \quad h_{2}(0)=-\frac{i}{\sqrt{p^{\prime}}} .
$$

Since $\xi$ is an analytic function of $\lambda_{1}$ and of $\lambda_{2}$,

$$
\left.\frac{d \xi}{d \lambda_{k}}\right|_{\lambda_{k}=0}=h_{k}(0) \neq 0, \quad k=1,2,
$$

the inverse functions $\lambda_{1}(\xi)$ and $\lambda_{2}(\xi)$ are analytic around zero. Set

$$
\lambda_{1,2}(\xi)=\xi \sum_{l=0}^{\infty} c_{l}^{(1,2)} \xi^{l}
$$

Substitute them in $d\left(\lambda_{1,2} ; \xi\right)=0$. We have

$$
\begin{aligned}
& \left(\xi \sum_{l=0}^{\infty} c_{l}^{(1,2)} \xi^{l}\right)^{2} q_{2}\left(\xi \sum_{l=0}^{\infty} c_{l}^{(1,2)} \xi^{l}\right)+g^{\prime} \xi^{3}\left(\sum_{l=0}^{\infty} c_{l}^{(1,2)} \xi^{l}\right) q_{1}\left(\xi \sum_{l=0}^{\infty} c_{l}^{(1,2)} \xi^{l}\right) \\
& +p^{\prime} \xi^{2} q_{2}\left(\xi \sum_{l=0}^{\infty} c_{l}^{(1,2)} \xi^{l}\right)=0 .
\end{aligned}
$$


Expanding the right-hand side in $\xi$ and setting the coefficients of $\xi^{2}$ and $\xi^{3}$ to be zeros, we arrive at

$$
\begin{gathered}
{\left[c_{0}^{(1,2)}\right]^{2}+p^{\prime}=0,} \\
2 c_{0}^{(1,2)} c_{1}^{(1,2)} q_{2}(0)+\left[c_{0}^{(1,2)}\right]^{3} q_{2}^{\prime}(0)+g^{\prime} c_{0}^{(1,2)} q_{1}(0)+p^{\prime} q_{2}^{\prime}(0) c_{0}^{(1,2)}=0 .
\end{gathered}
$$

Solve them to get

$$
\begin{aligned}
& c_{0}^{(1)}=-i \sqrt{p^{\prime}}, \quad c_{0}^{(2)}=i \sqrt{p^{\prime}}, \\
& c_{1}^{(1)}=c_{2}^{(1)}=-\frac{1}{2} g^{\prime} q_{1}(0) / q_{2}(0)=-\mu / 2,
\end{aligned}
$$

where the signs for $c_{0}^{(1)}$ and $c_{0}^{(2)}$ are chosen by (2.24). Substitute them into (2.25) and we have (2.18).

Next we claim that

$$
\operatorname{Re} \lambda_{k}(\xi) \neq 0, \quad k=1, \ldots, n, \quad \text { if } \quad \xi \neq 0 \text { is real. }
$$

If this is not true, set $\lambda_{k}(\xi)=i \omega$, with $\omega$ real. Substituting into $d\left(\lambda_{k} ; \xi\right)=0$ we have

$$
(i \omega)^{2} q_{2}(i \omega)+g^{\prime} \xi^{2} i \omega q_{1}(i \omega)+p^{\prime} \xi^{2} q_{2}(i \omega)=0 .
$$

Divided by $q_{2}(i \omega)$ and taking the imaginary part gives

$$
g^{\prime} \xi^{2} \omega \operatorname{Re}[L(a)(i \omega)]=0 .
$$

Since $g^{\prime}, \xi^{2}>0$ and (1.7), $\omega=0$. Then (2.27) becomes $p^{\prime} \xi^{2} q_{2}(0)=0$. This is impossible.

Equations (2.18), (2.23), (2.26) and the continuity of $\lambda_{k}(\xi)$ give us

$$
\begin{aligned}
& \operatorname{Re} \lambda_{1,2}(\xi)<0, \quad \xi \in \mathscr{R} \backslash\{0\}, \\
& \operatorname{Re} \lambda_{k}(\xi)<0, \quad k=3, \ldots, n, \quad \xi \in \mathscr{R} .
\end{aligned}
$$

As long as we can prove that there exist constants $\xi_{0}, \bar{\alpha}>0$, such that

$$
\operatorname{Re} \lambda_{k}(\xi) \leqq-\alpha, \quad k=1, \ldots, n, \quad \text { for } \xi \geqq \xi_{0} \quad \text { and } \xi \leqq-\xi_{0},
$$

then $(2.19)_{1}$ and $(2.19)_{2}$ follow easily.

If (2.28) is not true for some $1 \leqq k \leqq n$, then we can find a sequence $\xi_{n} \rightarrow \infty$, such that $\operatorname{Re} \lambda_{k}\left(\xi_{n}\right) \rightarrow 0$. Set $\lambda_{k}\left(\xi_{n}\right)=r_{n}+i \omega_{n}$, where $r_{n} \rightarrow 0$. Substitute it into $d\left(\lambda_{k}\left(\xi_{n}\right) ; \xi_{n}\right)=0$. We have

$$
\left(r_{n}+i \omega_{n}\right)^{2}+g^{\prime} \xi_{n}^{2}\left(r_{n}+i \omega_{n}\right) L(a)\left(r_{n}+i \omega_{n}\right)+p^{\prime} \xi_{n}^{2}=0,
$$

since $q_{2}\left(\lambda_{k}\left(\xi_{n}\right)\right) \neq 0$ by Lemma 2 .1. Separate the real and imaginary parts,

$$
\begin{gathered}
r_{n}^{2}-\omega_{n}^{2}+g^{\prime} \xi_{n}^{2}\left[r_{n} \operatorname{Re} L(a)-\omega_{n} \operatorname{Im} L(a)\right]_{r_{n}+i \omega_{n}}+p^{\prime} \xi_{n}^{2}=0 \\
2 r_{n} \omega_{n}+g^{\prime} \xi_{n}^{2}\left[r_{n} \operatorname{Im} L(a)+\omega_{n} \operatorname{Re} L(a)\right]_{r_{n}+i \omega_{n}}=0 .
\end{gathered}
$$

If $\omega_{n}$ is bounded, then there is a subsequence, denoted again by $\omega_{n}$, such that $\omega_{n} \rightarrow \omega_{0}$. Divide $(2.30)_{2}$ by $\xi_{n}^{2}$, and let $n \rightarrow \infty$. We get $g^{\prime} \omega_{0} \operatorname{Re} L(a)\left(i \omega_{0}\right)=0$. Then $\omega_{0}=0$ since $a$ is strongly positive definite. Divide $(2.30)_{1}$ by $\xi_{n}^{2}$, let $n \rightarrow \infty$, and note that $r_{n}, \omega_{n} \rightarrow 0$. We have $p^{\prime}=0$. This contradicts assumption (2.3).

Therefore $\omega_{n}$ is unbounded. There exists a subsequence, again denoted by $\omega_{n}$, such that $\omega_{n} \rightarrow \infty$. Divide (2.29) by $\xi_{n}^{2}$, let $n \rightarrow \infty$, and note that $\lim _{s \rightarrow \infty} s L(a)(s)=a(0)$ 
by properties of Laplace transform. We have

$$
\lim _{n \rightarrow \infty}\left(\frac{\omega_{n}}{\xi_{n}}\right)^{2}=f^{\prime}
$$

Solve (2.30) for $\operatorname{Re} L(a)$ and $\operatorname{Im} L(a)$. Especially,

$$
\operatorname{Re} L(a)\left(r_{n}+i \omega_{n}\right)=-\frac{r_{n}}{g^{\prime} \xi_{n}^{2}}-\frac{r_{n} p^{\prime}}{g^{\prime}\left(r_{n}^{2}+\omega_{n}^{2}\right)} .
$$

Using (2.31) and $r_{n} \rightarrow 0, \omega_{n} \rightarrow \infty$, we get

$$
\lim _{n \rightarrow \infty} \omega_{n}^{2} \operatorname{Re} L(a)\left(r_{n}+i \omega_{n}\right)=-\lim _{n \rightarrow \infty} \frac{r_{n}}{g^{\prime}}\left(\frac{\omega_{n}}{\xi_{n}}\right)^{2}-\lim _{n \rightarrow \infty} \frac{p^{\prime}}{g^{\prime}} \frac{r_{n} \omega_{n}^{2}}{r_{n}^{2}+\omega_{n}^{2}}=0 .
$$

Then the real part of $\lim _{n \rightarrow \infty}\left(r_{n}+i \omega_{n}\right) L(a)\left(r_{n}+i \omega_{n}\right)=a(0)$ gives us

$$
\lim _{n \rightarrow \infty} \omega_{n} \operatorname{Im} L(a)\left(r_{n}+i \omega_{n}\right)=-a(0) .
$$

By (2.32) and (2.33) we have

$$
\begin{aligned}
& \lim _{n \rightarrow \infty} \operatorname{Re}\left[\left(r_{n}+i \omega_{n}\right)^{2} L(a)\left(r_{n}+i \omega_{n}\right)\right] \\
& \quad=\lim _{n \rightarrow \infty}\left[\left(r_{n}^{2}-\omega_{n}^{2}\right) \operatorname{Re} L(a)\left(r_{n}+i \omega_{n}\right)-2 r_{n} \omega_{n} \operatorname{Im} L(a)\left(r_{n}+i \omega_{n}\right)\right] \\
& \quad=0 .
\end{aligned}
$$

On the other hand,

$$
L\left(a^{\prime \prime}\right)(s)=s^{2} L(a)(s)-s a(0)-a^{\prime}(0) .
$$

Set $s=r_{n}+i \omega_{n}$ and take real part. We arrive at

$$
\operatorname{Re} L\left(a^{\prime \prime}\right)\left(r_{n}+i \omega_{n}\right)-\operatorname{Re}\left[\left(\mathrm{r}_{n}+i \omega_{n}\right)^{2} L(a)\left(r_{n}+i \omega_{n}\right)\right]+r_{n} a(0)=-a^{\prime}(0) .
$$

Let $n \rightarrow \infty$. We get

$$
\lim _{n \rightarrow \infty} \operatorname{Re}\left[\left(r_{n}+i \omega_{n}\right)^{2} L(a)\left(r_{n}+i \omega_{n}\right)\right]=a^{\prime}(0)<0
$$

by (1.8). This is a contradiction to (2.34).

ii) Let $\lambda^{0}$ be a finite cluster point of $\lambda_{k}(\xi)$ as $\xi \rightarrow \infty$, i.e., there is a sequence $\xi_{n} \rightarrow \infty$ such that $\lambda_{k}\left(\xi_{n}\right) \rightarrow \lambda^{0} . d\left(\lambda_{k}\left(\xi_{n}\right) ; \xi_{n}\right)=0$ gives us

$$
\frac{1}{\xi_{n}^{2}} \lambda_{k}\left(\xi_{n}\right)^{2} q_{2}\left(\lambda_{k}\left(\xi_{n}\right)\right)+q\left(\lambda_{k}\left(\xi_{n}\right)\right)=0
$$

where $q$ is defined by (2.22). Let $n \rightarrow \infty$. We get $q\left(\lambda^{0}\right)=0$. As $\xi \rightarrow \infty$, either $\lambda_{k}(\xi) \rightarrow \lambda_{k}^{0}$ or $\lambda_{k}(\xi) \rightarrow \infty$, where $\lambda_{k}^{0}$ is a zero of $q$. If this is not true, $\lambda_{k}(\xi)$ has infinitely many finite cluster points since $\lambda_{k}$ is a continuous function of $\xi$, and all these cluster points are zeros of $q$. This is impossible because $q$ is a polynomial of degree $m_{2}$. If $\lim _{\xi \rightarrow \infty} \lambda_{k}(\xi)=\infty$, rewrite $d\left(\lambda_{k}(\xi) ; \xi\right)=0$ as

$$
\left[\frac{\lambda_{k}(\xi)}{\xi}\right]^{2}+g^{\prime} \lambda_{k}(\xi) L(a)\left(\lambda_{k}(\xi)\right)+p^{\prime}=0
$$


Let $\xi \rightarrow \infty$. Note that $\lim _{s \rightarrow \infty} s L(a)(s)=a(0)$. We have $\lim _{\xi \rightarrow \infty}\left[\lambda_{k}(\xi) / \xi\right]^{2}=-f^{\prime}$. It is easy to see by argument principle, for example, that for an $m$-zero of $q$ there are exactly $m \lambda_{k}(\xi)$ 's approaching it as $\xi \rightarrow \infty$. Therefore we have (2.20) and (2.21). $\lambda_{1}(\xi)$ and $\lambda_{2}(\xi)$ are complex conjugates since $d(s ; \xi)$ has real coefficients. Q.E.D

Lemma 2.4. Assume that the hypotheses of Lemma 2.1 are in force and $\xi$ is real. Then for small $\delta>0$, there exists a constant $C=C(\delta)>0$, such that

i) for $|\xi| \leqq \delta$,

$$
\begin{gathered}
\frac{\lambda_{1,2} q_{2}\left(\lambda_{1,2}\right)}{d^{\prime}\left(\lambda_{1,2} ; \xi\right)}=\frac{1}{2}+O(\xi), \\
\frac{i \xi q_{2}\left(\lambda_{1,2}\right)}{d^{\prime}\left(\lambda_{1,2} ; \xi\right)}=\mp \frac{1}{2 \sqrt{p^{\prime}}}+O(\xi), \\
\frac{i \xi\left[g^{\prime} \lambda_{1,2} q_{1}\left(\lambda_{1,2}\right)+p^{\prime} q_{2}\left(\lambda_{1,2}\right)\right]}{d^{\prime}\left(\lambda_{1,2} ; \xi\right)}=\mp \frac{\sqrt{p^{\prime}}}{2}+O(\xi)
\end{gathered}
$$

and

$$
\begin{gathered}
\left|\sum_{k=3}^{n} \frac{\lambda_{k} q_{2}\left(\lambda_{k}\right)}{d^{\prime}\left(\lambda_{k} ; \xi\right)} e^{\lambda_{k} t}\right|,\left|\sum_{k=3}^{n} \frac{i \xi q_{2}\left(\lambda_{k}\right)}{d^{\prime}\left(\lambda_{k} ; \xi\right)} e^{\lambda_{k} t}\right|, \\
\left|\sum_{k=3}^{n} \frac{i \xi\left[g^{\prime} \lambda_{k} q_{1}\left(\lambda_{k}\right)+p^{\prime} q_{2}\left(\lambda_{k}\right)\right]}{d^{\prime}\left(\lambda_{k} ; \xi\right)} e^{\lambda_{k} t}\right| \leqq C e^{-t / C}, \quad t \geqq 0 ;
\end{gathered}
$$

ii) for $|\xi| \geqq \delta$,

$$
\begin{gathered}
\left|\sum_{k=1}^{n} \frac{\lambda_{k} q_{2}\left(\lambda_{k}\right)}{d^{\prime}\left(\lambda_{k} ; \xi\right)} e^{\lambda_{k} t}\right|,\left|\sum_{k=1}^{n} \frac{i \xi q_{2}\left(\lambda_{k}\right)}{d^{\prime}\left(\lambda_{k} ; \xi\right)} e^{\lambda_{k} t}\right|, \\
\left|\sum_{k=1}^{n} \frac{i \xi\left[g^{\prime} \lambda_{k} q_{1}\left(\lambda_{k}\right)+p^{\prime} q_{2}\left(\lambda_{k}\right)\right]}{d^{\prime}\left(\lambda_{k} ; \xi\right)} e^{\lambda_{k} t}\right| \leqq C e^{-t / C}, \quad t \geqq 0 .
\end{gathered}
$$

Proof. i) Using (2.18) and the expression for $d^{\prime}\left(\lambda_{k} ; \xi\right)$ in (2.13), (2.35) can be checked by direct computation. The proof of (2.36) is similar to that of (2.37) below.

ii) We only estimate the first term in (2.37). The other terms can be done in exactly the same way.

From Lemma 2.1 we know that $d^{\prime}\left(\lambda_{k}(\xi) ; \xi\right) \neq 0$ except of a finite number of $\xi$ 's (including $\xi=0$ ). Suppose that $d^{\prime}\left(\lambda_{k}(\xi) ; \xi\right) \neq 0, k=1, \ldots, n$, on a finite interval $\left[\xi_{1}, \xi_{2}\right]$. Then on such an interval (2.37) follows easily from the continuity of $\lambda_{k}$ on $\xi$ and (2.19). Therefore all we need to do is to prove (2.37) for $\xi$ in a small neighborhood of $\xi_{0}$ with $d^{\prime}\left(\lambda_{k}\left(\xi_{0}\right) ; \xi_{0}\right)=0$ for some $k$ and for $\xi \rightarrow \infty$.

Consider a small neighborhood of $\xi_{0}$ in which $d^{\prime}\left(\lambda_{k}\left(\xi_{0}\right) ; \xi_{0}\right)=0$ for some $1 \leqq k \leqq n$ and $d^{\prime}\left(\lambda_{k}(\xi) ; \xi\right) \neq 0$ for $\xi \neq \xi_{0}, k=1, \ldots, n$. For definiteness let's assume that $\lambda_{0} \equiv$ $\lambda_{1}\left(\xi_{0}\right)=\lambda_{2}\left(\xi_{0}\right)=\cdots=\lambda_{m}\left(\xi_{0}\right), 2 \leqq m \leqq n$, and $\lambda_{k}\left(\xi_{0}\right) \neq \lambda_{0}, k=m+1, \ldots, n$. By the continuity of $\lambda_{k}$, we can choose $0<\varepsilon_{0} \leqq \alpha / 4$ and $\delta_{0}>0$, where $\alpha$ is the constant in Lemma 2.3 , such that for $\left|\xi-\xi_{0}\right|<\delta_{0}$,

$$
\begin{array}{ll}
\left|\lambda_{k}(\xi)-\lambda_{0}\right|<\varepsilon_{0}, & k=1, \ldots, m, \\
\left|\lambda_{k}(\xi)-\lambda_{0}\right|>2 \varepsilon_{0}, & k=m+1, \ldots, n .
\end{array}
$$


Using residue theorem, for any fixed $\xi, 0<\left|\xi-\xi_{0}\right|<\delta_{0}$,

$$
\sum_{k=1}^{m} \frac{\lambda_{k} q_{2}\left(\lambda_{k}\right)}{d^{\prime}\left(\lambda_{k} ; \xi\right)} e^{\lambda_{k} t}=\frac{1}{2 \pi i} \oint_{\left|\lambda-\lambda_{0}\right|=(3 / 2) \varepsilon_{0}} \frac{\lambda q_{2}(\lambda)}{d(\lambda ; \xi)} e^{\lambda t} d \lambda .
$$

Using (2.38) and (2.19), we have for $\left|\lambda-\lambda_{0}\right|=\frac{3}{2} \varepsilon_{0}$,

$$
\begin{aligned}
& |d(\lambda ; \xi)|=\left|\lambda-\lambda_{1}(\xi)\right| \cdot\left|\lambda-\lambda_{2}(\xi)\right| \cdots\left|\lambda-\lambda_{n}(\xi)\right|>\left(\varepsilon_{0} / 2\right)^{n}, \\
& \operatorname{Re} \lambda=\operatorname{Re}\left(\lambda-\lambda_{0}\right)+\operatorname{Re} \lambda_{0} \leqq\left|\lambda-\lambda_{0}\right|+\operatorname{Re} \lambda_{1}\left(\xi_{0}\right)<-\alpha / 2 .
\end{aligned}
$$

Therefore

$$
\left|\sum_{k=1}^{m} \frac{\lambda_{k} q_{2}\left(\lambda_{k}\right)}{d^{\prime}\left(\lambda_{k} ; \xi\right)} e^{\lambda_{k} t}\right| \leqq \frac{\frac{3}{2} \varepsilon_{0} \max _{\left|\lambda-\lambda_{0}\right|=(3 / 2) \varepsilon_{0}}\left|\lambda q_{2}(\lambda)\right| e^{t \operatorname{Re} \lambda}}{\left(\varepsilon_{0} / 2\right)^{n}} \leqq C e^{-(\alpha / 2) t}
$$

Similarly we can get the same estimate for $\left|\sum_{k=m+1}^{n} \lambda_{k} q_{2}\left(\lambda_{k}\right) / d^{\prime}\left(\lambda_{k} ; \xi\right) e^{\lambda_{k} t}\right|$. Equation (2.37) holds in a small neighborhood of $\xi_{0}$.

Next consider the case when $\xi \rightarrow \infty$,

$$
\left|\sum_{k=1}^{n} \frac{\lambda_{k} q_{2}\left(\lambda_{k}\right)}{d^{\prime}\left(\lambda_{k} ; \xi\right)} e^{\lambda_{k} t}\right| \leqq\left|\frac{\lambda_{1} q_{2}\left(\lambda_{1}\right)}{d^{\prime}\left(\lambda_{1} ; \xi\right)}\right| e^{t \operatorname{Re} \lambda_{1}}+\left|\frac{\lambda_{2} q_{2}\left(\lambda_{2}\right)}{d^{\prime}\left(\lambda_{2} ; \xi\right)}\right| e^{t \operatorname{Re} \lambda_{2}}+\left|\sum_{k=3}^{n} \frac{\lambda_{k} q_{2}\left(\lambda_{k}\right)}{d^{\prime}\left(\lambda_{k} ; \xi\right)} e^{\lambda_{k} t}\right| .
$$

Note that

$$
\begin{aligned}
\frac{\lambda_{1} q_{2}\left(\lambda_{1}\right)}{d^{\prime}\left(\lambda_{1} ; \xi\right)} & =\frac{\lambda_{1}(\xi) q_{2}\left(\lambda_{1}(\xi)\right)}{\left(\lambda_{1}(\xi)-\lambda_{2}(\xi)\right)\left(\lambda_{1}(\xi)-\lambda_{3}(\xi)\right) \cdots\left(\lambda_{1}(\xi)-\lambda_{n}(\xi)\right)} \\
& =\frac{1}{1-\lambda_{2}(\xi) / \lambda_{1}(\xi)} \frac{q_{2}\left(\lambda_{1}(\xi)\right)}{\left(\lambda_{1}(\xi)-\lambda_{3}(\xi)\right) \cdots\left(\lambda_{1}(\xi)-\lambda_{n}(\xi)\right)}
\end{aligned}
$$

and $q_{2}$ is a polynomial of degree $m_{2}=n-2$. Using (2.20) and (2.21) we conclude that $\lim _{\xi \rightarrow \infty} \lambda_{1} q_{2}\left(\lambda_{1}\right) / d^{\prime}\left(\lambda_{1} ; \xi\right)=1 / 2$. Together with (2.19), the first term on the righthand side of (2.39) is bounded by $C e^{-t / C}$ for large $\xi$. Similarly we have the same bound for the second term. The third term can be estimated in the same way if $q(s)$ defined by (2.22) has only simple zeros. If $q(s)$ has a double zero, then we use the same technique as in the case above when $\xi$ is in a small neighborhood of $\xi_{0}$, replacing $\lambda_{0}$ by the double zero of $q$ and considering large $\xi$. Q.E.D

Theorem 2.5. Assume that (2.3) and (1.6) hold, $U_{0} \in L^{1}(\mathscr{R}) \cap H^{s}(\mathscr{R}), D^{-1} \phi \in C([0, \infty)$; $\left.H^{s+1}(\mathscr{R})\right) \cap C\left([0, \infty) ; W^{l+1, p}(\mathscr{R})\right)$, where $s \geqq 2,-1 \leqq l \leqq s$ and $p \in[1,2]$. Then the solution $U$ of $(2.1),(2.2)$ satisfies

$$
\begin{aligned}
\left\|D^{k} U\right\|(t) \leqq & C\left[(1+t)^{-1 / 2(k+(1 / 2))}\left\|U_{0}\right\|_{L^{1}}+e^{-t / C}\left\|D^{k} U_{0}\right\|\right. \\
& +\int_{0}^{t}(1+t-\tau)^{-1 / 2(k-l+1 / p-1 / 2)}\left\|D^{l} \phi\right\|_{L^{p}}(\tau) d \tau \\
& \left.+\int_{0}^{t} e^{-(t-\tau) / C}\left\|D^{k} \phi\right\|(\tau) d \tau\right]
\end{aligned}
$$

for $\max (0, l) \leqq k \leqq s$, where $t \geqq 0$, and $C>0$ is a constant. 
Proof. By Lemmas 2.2, 2.3 and 2.4, for a small $\delta>0$ and real $\xi$, if $|\xi| \leqq \delta$,

$$
\begin{aligned}
\left|U^{\wedge}(\xi, t)\right| \leqq & C\left|U_{0}^{\wedge}(\xi)\right|\left[e^{-(\mu / 4) \xi^{2} t}+e^{-t / C}\right] \\
& +C \int_{0}^{t}\left[e^{-(\mu / 4) \xi^{2}(t-\tau)}+e^{-(t-\tau) / C}\right]\left|\phi^{\wedge}(\xi, \tau)\right| d \tau
\end{aligned}
$$

if $|\xi| \geqq \delta$,

$$
\left|U^{\wedge}(\xi, t)\right| \leqq C\left|U_{0}^{\wedge}(\xi)\right| e^{-t / C}+C \int_{0}^{t} e^{-(t-\tau) / C}\left|\phi^{\wedge}(\xi, \tau)\right| d \tau
$$

Note that $\left(D^{k} U\right)^{\wedge}(\xi, t)$ satisfies $(2.4),(2.5)$ with $\phi^{\wedge}$ being replaced by $(i \xi)^{k} \phi^{\wedge}$ and $U_{\hat{0}}$ by $(i \xi)^{k} U_{0}^{\wedge}$. Therefore

$$
\begin{aligned}
& \left\|D^{k} U\right\|^{2}(t)=\left\|\left(D^{k} U\right)^{\wedge}\right\|^{2}(t) \\
& =\left(\int_{|\xi| \leqq \delta}+\int_{|\xi| \geqq \delta}\right)\left|\left(D^{k} U\right)^{\wedge}\right|^{2}(\xi, t) d \xi \\
& \leqq C \int_{|\xi| \leqq \delta}\left|(i \xi)^{k} U_{0}^{\wedge}(\xi)\right|^{2}\left[e^{-(\mu / 2) \xi^{2} t}+e^{-t / C}\right] d \xi+C \int_{|\xi| \geqq \delta}\left|(i \xi)^{k} U_{0}^{\wedge}(\xi)\right|^{2} e^{-t / C} d \xi \\
& +C \int_{|\xi| \leqq \delta}\left\{\int_{0}^{t}\left[e^{-(\mu / 4) \xi^{2}(t-\tau)}+e^{-(t-\tau) / C}\right]\left|(i \xi)^{k} \phi^{\wedge}(\xi, \tau)\right| d \tau\right\}^{2} d \xi \\
& +C \int_{|\xi| \geqq \delta}\left\{\int_{0}^{t} e^{-(t-\tau) / C}\left|(i \xi)^{k} \phi^{\wedge}(\xi, \tau)\right| d \tau\right\}^{2} d \xi \\
& \leqq C \int_{|\xi| \leqq \delta}\left|\xi^{k} U_{0}^{\wedge}(\xi)\right|^{2} e^{-(\mu / 2) \xi^{2} t} d \xi+C\left\|\left(D^{k} U_{0}\right)^{\wedge}\right\|^{2} e^{-t / C} \\
& +C\left\{\int_{0}^{t}\left[\int_{|\xi| \leqq \delta}\left(e^{-(\mu / 4) \xi^{2}(t-\tau)}+e^{-(t-\tau) / C}\right)^{2}\left|\xi^{k} \phi^{\wedge}(\xi, \tau)\right|^{2} d \xi\right]^{1 / 2} d \tau\right\}^{2} \\
& +C\left\{\int_{0}^{t}\left[\int_{|\xi| \geqq \delta} e^{-(t-\tau) / C}\left|\xi^{k} \phi^{\wedge}(\xi, \tau)\right|^{2} d \xi\right]^{1 / 2} d \tau\right\}^{2} \\
& \leqq C\left\|U_{0}^{\wedge}\right\|_{L^{\infty}}^{2}(1+t)^{-k-(1 / 2)}+C\left\|\left(D^{k} U_{0}\right)^{\wedge}\right\|^{2} e^{-t / C} \\
& +C\left\{\int_{0}^{t}\left[\int_{|\xi| \leqq \delta}|\xi|^{2(k-l)} e^{-(\mu / 2) \xi^{2}(t-\tau)}\left|\left(D^{l} \phi\right)^{\wedge}(\xi, \tau)\right|^{2} d \xi\right]^{1 / 2} d \tau\right\}^{2} \\
& +C\left\{\int_{0}^{t} e^{-(t-\tau) / C}\left\|\left(D^{k} \phi\right)^{\wedge}\right\|(\tau) d \tau\right\}^{2} \\
& \leqq C\left\|U_{0}^{\wedge}\right\|_{L^{\infty}}^{2}(1+t)^{-k-(1 / 2)}+C\left\|\left(D^{k} U_{0}\right)^{\wedge}\right\|^{2} e^{-t / C} \\
& +C\left\{\int_{0}^{t}(1+t-\tau)^{-1 / 2(k-l)-1 / 4\left(1-\left(2 / p^{\prime}\right)\right)}\left\|\left(D^{l} \phi\right)^{\wedge}\right\|_{L^{p^{\prime}}}(\tau) d \tau\right\}^{2} \\
& +C\left\{\int_{0}^{t} e^{-(t-\tau) / C}\left\|\left(D^{k} \phi\right)^{\wedge}\right\|(\tau) d \tau\right\}^{2} \\
& \leqq C\left[\left\|U_{0}\right\|_{L^{1}}^{2}(1+t)^{-(k+(1 / 2))}+\left\|D^{k} U_{0}\right\|^{2} e^{-t / C}\right]
\end{aligned}
$$




$$
\begin{aligned}
& +C\left\{\int_{0}^{t}(1+t-\tau)^{-1 / 2(k-l+(1 / p)-(1 / 2))}\left\|D^{l} \phi\right\|_{L^{p}}(\tau) d \tau\right\}^{2} \\
& +C\left\{\int_{0}^{t} e^{-(t-\tau) / C}\left\|D^{k} \phi\right\|(\tau) d \tau\right\}^{2},
\end{aligned}
$$

where we have used the Hausdorff-Young inequality $\left\|w^{\wedge}\right\|_{L^{p^{\prime}}} \leqq C\|w\|_{L^{p}}, p \in[1,2]$, $1 / p+1 / p^{\prime}=1$. Taking square root gives us (2.40). Q.E.D

To obtain the decay estimate for the difference between the solution of (1.1) and the solution of (1.13), again we need to know the decay rate for the difference between the solutions of the linearizations.

Note that $\mu$ defined by (2.17) is positive. We consider the following parabolic system which is the linearization of (1.13):

$$
\left\{\begin{array}{l}
\tilde{u}_{t}-\tilde{v}_{x}=\frac{\mu}{2} \tilde{u}_{x x} \\
\tilde{v}_{t}-p^{\prime} \tilde{u}_{x}=\frac{\mu}{2} \tilde{v}_{x x}+\tilde{\phi}, \quad x \in \mathscr{R}, \quad t>0, \\
\tilde{U}(x, 0)=U_{0}(x), \quad x \in \mathscr{R},
\end{array}\right.
$$

where $\tilde{U} \equiv(\tilde{u}, \tilde{v})$. Take Fourier transform,

$$
\left\{\begin{array}{c}
\tilde{u}_{t}^{\wedge}-i \xi \tilde{v}^{\wedge}=\frac{\mu}{2}(i \xi)^{2} \tilde{u}^{\wedge} \\
\tilde{v}_{t}^{\wedge}-p^{\prime} i \xi \tilde{u}^{\wedge}=\frac{\mu}{2}(i \xi)^{2} \tilde{v}^{\wedge}+\tilde{\phi}^{\wedge} \\
\tilde{U}^{\wedge}(\xi, 0)=U_{0}^{\wedge}(\xi) .
\end{array}\right.
$$

Solve these ODE's,

$$
\begin{aligned}
\tilde{u}^{\wedge}(\xi, t)= & u_{0}^{\wedge}(\xi)\left\{\frac{1}{2} e^{-i \xi \sqrt{p^{\prime} t}-(\mu / 2) \xi^{2} t}+\frac{1}{2} e^{i \xi \sqrt{p^{\prime} t}-(\mu / 2) \xi^{2} t}\right\} \\
& +v_{0}^{\wedge}(\xi)\left\{-\frac{1}{2 \sqrt{p^{\prime}}} e^{-i \xi \sqrt{p^{\prime} t}-(\mu / 2) \xi^{2} t}+\frac{1}{2 \sqrt{p^{\prime}}} e^{i \xi \sqrt{p^{\prime} t}-(\mu / 2) \xi^{2} t}\right\} \\
& +\int_{0}^{t}\left[-\frac{1}{2 \sqrt{p^{\prime}}} e^{-i \xi \sqrt{p^{\prime}}(t-\tau)-(\mu / 2) \xi^{2}(t-\tau)}+\frac{1}{2 \sqrt{p^{\prime}}} e^{i \xi \sqrt{p^{\prime}}(t-\tau)-(\mu / 2) \xi^{2}(t-\tau)}\right] \tilde{\phi}^{\wedge}(\xi, \tau) d \tau \\
\tilde{v}^{\wedge}(\xi, t)= & u_{0}^{\wedge}(\xi)\left\{-\frac{\sqrt{p^{\prime}}}{2} e^{-i \xi \sqrt{p^{\prime} t}-(\mu / 2) \xi^{2} t}+\frac{\sqrt{p^{\prime}}}{2} e^{i \xi \sqrt{p^{\prime} t}-(\mu / 2) \xi^{2} t}\right\} \\
& +v_{0}^{\wedge}(\xi)\left\{\frac{1}{2} e^{-i \xi \sqrt{p^{\prime} t}-(\mu / 2) \xi^{2} t}+\frac{1}{2} e^{i \xi \sqrt{p^{\prime} t}-(\mu / 2) \xi^{2} t}\right\} \\
& +\int_{0}^{t}\left[\frac{1}{2} e^{-i \xi \sqrt{p^{\prime}}(t-\tau)-(\mu / 2) \xi^{2}(t-\tau)}+\frac{1}{2} e^{i \xi \sqrt{p^{\prime}}(t-\tau)-(\mu / 2) \xi^{2}(t-\tau)}\right] \tilde{\phi}^{\wedge}(\xi, \tau) d \tau .
\end{aligned}
$$


Theorem 2.6. Assume that (2.3) and (1.6) hold, $U_{0} \in L^{1}(\mathscr{R}) \cap H^{s}(\mathscr{R}), D^{-1} \phi, D^{-1} \tilde{\phi} \in$ $C\left([0, \infty) ; H^{s+1}(\mathscr{R})\right) \cap C\left([0, \infty) ; W^{l+1, p}(\mathscr{R})\right), D^{-1} \tilde{\phi} \in C\left([0, \infty) ; W^{l^{\prime}+1, q}(\mathscr{R})\right)$, where $s \geqq 2,-1 \leqq l, l^{\prime} \leqq s-1$ and $p, q \in[1,2]$. Then the solution $U$ of $(2.1),(2.2)$ and the solution $\tilde{U}$ of $(2.41),(2.42)$ satisfy

$$
\begin{aligned}
\left\|D^{k}(U-\tilde{U})\right\|(t) \leqq & C\left[(1+t)^{-1 / 2(k+(3 / 2))}\left\|U_{0}\right\|_{L^{1}}+e^{-t / C}\left\|U_{0}\right\|_{k+1}\right. \\
& +\int_{0}^{t}(1+t-\tau)^{-1 / 2(k-l+(1 / p)-(1 / 2))}\left\|D^{l}(\phi-\tilde{\phi})\right\|_{L^{p}}(\tau) d \tau \\
& +\int_{0}^{t}(1+t-\tau)^{-1 / 2\left(k-l^{\prime}+(1 / q)+(1 / 2)\right)}\left\|D^{l^{\prime}} \tilde{\phi}\right\|_{L^{q}}(\tau) d \tau \\
& \left.+\int_{0}^{t} e^{-(t-\tau) / C}\left(\left\|D^{k} \phi\right\|+\left\|D^{k} \tilde{\phi}\right\|_{1}\right)(\tau) d \tau\right]
\end{aligned}
$$

for $\max \left(0, l, l^{\prime}\right) \leqq k \leqq s-1$, where $t \geqq 0$, and $C>0$ is a constant.

Proof. By Lemmas 2.2, 2.3, 2.4 and (2.43), for a small $\delta>0$ and real $\xi$, if $|\xi| \leqq \delta$,

$$
\begin{aligned}
\left|U^{\wedge}-\tilde{U}^{\wedge}\right|(\xi, \tau) \leqq & C\left|U_{0}^{\wedge}(\xi)\right|\left[|\xi| e^{-(\mu / 4) \xi^{2} t}+e^{-t / C}\right] \\
& +C \int_{0}^{t}\left[e^{-(\mu / 4) \xi^{2}(t-\tau)}\left|\phi^{\wedge}-\tilde{\phi}^{\wedge}\right|(\xi, \tau)+e^{-(t-\tau) / C}\left|\phi^{\wedge}(\xi, \tau)\right|\right. \\
& \left.+|\xi| e^{-(\mu / 4) \xi^{2}(t-\tau)}\left|\tilde{\phi}^{\wedge}(\xi, \tau)\right|\right] d \tau,
\end{aligned}
$$

if $|\xi| \geqq \delta$,

$$
\begin{aligned}
\left|U^{\wedge}-\tilde{U}^{\wedge}\right|(\xi, \tau) & \leqq\left|U^{\wedge}(\xi, \tau)\right|+\left|\tilde{U}^{\wedge}(\xi, \tau)\right| \\
& \leqq C\left\{e^{-t / C}\left|U_{0}^{\wedge}(\xi)\right|+\int_{0}^{t} e^{-(t-\tau) / C}\left[\left|\phi^{\wedge}(\xi, \tau)\right|+\left|\tilde{\phi}^{\wedge}(\xi, \tau)\right|\right] d \tau\right\} .
\end{aligned}
$$

Theorem 2.6 then follows in exactly the same way as in the proof of Theorem 2.5 .

Q.E.D

\section{Energy Estimates}

Consider the following Cauchy problem

$$
\begin{gathered}
w_{t t}(x, t)=f\left(w_{x}(x, t)\right)_{x}+\int_{0}^{t} a^{\prime}(t-\tau) g\left(w_{x}(x, \tau)\right)_{x} d \tau+\phi(x, t), \quad x \in \mathscr{R}, \quad t>0, \\
w(x, 0)=w_{0}(x), \quad w_{t}(x, 0)=w_{1}(x), \quad x \in \mathscr{R} .
\end{gathered}
$$

Hrusa and Nohel [5] established the existence and uniqueness of the solution for (3.1), (3.2) and gave an energy estimate for the derivatives of the solution up to the third order. In this section we generalize their result to higher derivatives, which is needed later. Precisely, we have

Theorem 3.1. Assume that (1.5) and (1.6) $)_{1}$ hold. For each given $w_{0}, w_{1}$, and $\phi$ 
satisfying

$$
\begin{aligned}
& w_{0} \in L_{\mathrm{loc}}^{2}(\mathscr{R}), \quad w_{0}^{\prime}, w_{1} \in H^{s+2}(\mathscr{R}), \\
& \phi \in L^{1}\left([0, \infty) ; L^{2}(\mathscr{R})\right) \cap C\left([0, \infty) ; H^{s+1}(\mathscr{R})\right) \cap L^{\infty}\left([0, \infty) ; H^{s+1}(\mathscr{R})\right), \\
& \phi_{x} \in L^{2}\left([0, \infty) ; H^{s}(R)\right), \\
& \phi_{t} \in C\left([0, \infty) ; H^{s}(\mathscr{R})\right) \cap L^{\infty}\left([0, \infty) ; H^{s}(\mathscr{R})\right) \cap L^{2}\left([0, \infty) ; H^{s+1}(\mathscr{R})\right),
\end{aligned}
$$

where $s \geqq 0$, if

$$
\begin{aligned}
N_{s} \equiv & \left\|w_{0}^{\prime}\right\|_{s+2}^{2}+\left\|w_{1}\right\|_{s+2}^{2}+\sup _{t \geqq 0}\left\{\|\phi\|_{s+1}^{2}(t)+\left\|\phi_{t}\right\|_{s}^{2}(t)\right\} \\
& +\int_{0}^{\infty}\left[\|\phi\|_{s+1}^{2}+\left\|\phi_{t}\right\|_{s+1}^{2}\right](\tau) d \tau+\left[\int_{0}^{\infty}\|\phi\|(\tau) d \tau\right]^{2}
\end{aligned}
$$

is small, then the initial value problem (3.1), (3.2) has a unique solution $w$ defined on $\mathscr{R} \times[0, \infty)$ with

$$
\begin{aligned}
& w \in C\left([0, \infty) ; L_{\mathrm{ioc}}^{2}(\mathscr{R})\right), \\
& w_{x}, w_{t} \in C\left([0, \infty) ; H^{s+2}(\mathscr{R})\right) \cap L^{\infty}\left([0, \infty) ; H^{s+2}(\mathscr{R})\right), \\
& w_{t t} \in C\left([0, \infty) ; H^{s+1}(\mathscr{R})\right) \cap L^{\infty}\left([0, \infty) ; H^{s+1}(\mathscr{R})\right), \\
& w_{t t t} \in C\left([0, \infty) ; H^{s}(\mathscr{R})\right) \cap L^{\infty}\left([0, \infty) ; H^{s}(\mathscr{R})\right), \\
& w_{x x}, w_{t x}, w_{t t} \in L^{2}\left([0, \infty) ; H^{s+1}(\mathscr{R})\right), \\
& w_{t t t} \in L^{2}\left([0, \infty) ; H^{s}(\mathscr{R})\right),
\end{aligned}
$$

and

$$
\begin{aligned}
& \max _{0 \leqq \tau \leqq t}\left\{\left\|w_{x}\right\|_{s+2}^{2}+\left\|w_{t}\right\|_{s+2}^{2}+\left\|w_{t t}\right\|_{s+1}^{2}+\left\|w_{t t t}\right\|_{s}^{2}\right\}(\tau) \\
& \quad+\int_{0}^{t}\left\{\left\|w_{x x}\right\|_{s+1}^{2}+\left\|w_{t x}\right\|_{s+1}^{2}+\left\|w_{t t}\right\|_{s+1}^{2}+\left\|w_{t t t}\right\|_{s}^{2}\right\}(\tau) d \tau \leqq C N_{s}, \quad \forall t \geqq 0,
\end{aligned}
$$

where $C>0$ is a global constant.

The proof of this theorem is totally parallel to that of Theorem 1.1 in [5], which is a special case of $s=0$. Actually higher derivatives cause no difficulty. We can simply take derivatives with respect to $x$ at each step for getting $a$ priori estimate in [5]. This gives us the energy estimate (3.3) under the assumption that the left-hand side of (3.3) is small. Then using a local existence result which is a generalization of Lemma 2.1 in [5] we get the global result. We omit the detail.

To our purpose here, let's apply Theorem 3.1 to the problem (1.1), (1.2), with

$$
\begin{gathered}
w_{x}=u-\bar{u}, \quad w_{t}=v-\bar{v}, \\
\phi=\int_{-\infty}^{0} a^{\prime}(t-\tau) g(\eta(x, \tau))_{x} d \tau .
\end{gathered}
$$

We also assume $(1.6)_{2}$ so that (1.9) holds.

Theorem 3.2. Assume that (1.5) and (1.6) hold. For each given $\eta$ and $v_{0}$ satisfying 


$$
\begin{aligned}
& v_{0}-\bar{v} \in H^{s}(\mathscr{R}), \\
& \eta(\cdot, 0)-\bar{u} \in L^{1}(\mathscr{R}), \\
& \eta-\bar{u} \in L^{\infty}\left((-\infty, 0] ; H^{s}(\mathscr{R})\right) \cap C\left((-\infty, 0] ; H^{s}(\mathscr{R})\right),
\end{aligned}
$$

where $s \geqq 2$, if

$$
\hat{E}_{s} \equiv\left\|v_{0}-\bar{v}\right\|_{s}+\sup _{\tau \leqq 0}\|\eta-\bar{u}\|_{s}(\tau)
$$

is small, then the problem (1.1),(1.2) has a unique solution $U$ defined on $\mathscr{R} \times[0, \infty)$, with

$$
\begin{aligned}
& U-\bar{U} \in C\left([0, \infty) ; H^{s}(\mathscr{R})\right) \cap C^{1}\left([0, \infty) ; H^{s-1}(\mathscr{R})\right), \\
& U_{x} \in L^{2}\left([0, \infty) ; H^{s-1}(\mathscr{R})\right),
\end{aligned}
$$

and

$$
\sup _{0 \leqq \tau \leqq t}\|U-\bar{U}\|_{s}^{2}(\tau)+\int_{0}^{t}\left\|U_{x}\right\|_{s-1}^{2}(\tau) d(\tau) \leqq C \hat{E}_{s}^{2}
$$

where $C>0$ is a constant independent of $t$.

\section{Proofs of Theorems 1.1 and 1.4}

Once we have Theorems 2.5, 2.6 and the energy estimate (3.5), the proofs of Theorems 1.1 and 1.4 become a routine. We outline them as the following.

Proof of Theorem 1.1. Linearize (1.1) around the constant state $\bar{U}$,

$$
\left\{\begin{array}{l}
(u-\bar{u})_{t}-(v-\bar{v})_{x}=0 \\
(v-\bar{v})_{t}-f^{\prime}(\bar{u})(u-\bar{u})_{x}=g^{\prime}(\bar{u}) \int_{0}^{t} a^{\prime}(t-\tau)(u-\bar{u})_{x}(x, \tau) d \tau+h_{x}+\phi,
\end{array}\right.
$$

where

$$
\begin{aligned}
& h=f(u)-f(\bar{u})-f^{\prime}(\bar{u})(u-\bar{u})+\int_{0}^{t} a^{\prime}(t-\tau)\left[g(u)-g(\bar{u})-g^{\prime}(\bar{u})(u-\bar{u})\right](x, \tau) d \tau, \\
& \phi=\int_{-\infty}^{0} a^{\prime}(t-\tau) g(\eta)_{x}(x, \tau) d \tau .
\end{aligned}
$$

Use Theorem 2.5 , for $0 \leqq k \leqq j \leqq s-2-k$,

$$
\begin{aligned}
\left\|D^{j}(U-\bar{U})\right\|(t) \leqq & C\left\{(1+t)^{-1 / 2(j+(1 / 2))}\left\|U_{0}-\bar{U}\right\|_{L^{1}}+e^{-t / C}\left\|D^{j}\left(U_{0}-\bar{U}\right)\right\|\right. \\
& +\int_{0}^{t / 2}(1+t-\tau)^{-1 / 2(j+(3 / 2))}\|h\|_{L^{1}}(\tau) d \tau \\
& +\int_{t / 2}^{t}(1+t-\tau)^{-1 / 2(j-k+(3 / 2))}\left\|D^{k} h\right\|_{L^{1}}(\tau) d \tau \\
& +\int_{0}^{t} e^{-(t-\tau) / C}\left\|D^{j+1} h\right\|(\tau) d \tau+\int_{0}^{t}(1+t-\tau)^{-1 / 2(j+1)}\left\|D^{-1} \phi\right\|(\tau) d \tau \\
& \left.+\int_{0}^{t} e^{-(t-\tau) / C}\left\|D^{j} \phi\right\|(\tau) d \tau\right\} .
\end{aligned}
$$


Sum' up $j$,

$$
\begin{aligned}
& \left\|D^{k}(U-\bar{U})\right\|_{s-2-2 k}(t) \\
& \leqq \\
& \quad C\left\{(1+t)^{-1 / 2(k+(1 / 2))} E_{s}+\int_{0}^{t / 2}(1+t-\tau)^{-1 / 2(k+(3 / 2))}\|h\|_{L^{1}}(\tau) d \tau\right. \\
& \quad+\int_{t / 2}^{t}(1+t-\tau)^{-3 / 4}\left\|D^{k} h\right\|_{L^{1}}(\tau) d \tau+\int_{0}^{t} e^{-(t-\tau) / C}\left\|D^{k+1} h\right\|_{s-2-2 k}(\tau) d \tau \\
& \left.\quad+\int_{0}^{t}(1+t-\tau)^{-1 / 2(k+1)}\left\|D^{-1} \phi\right\|(\tau) d \tau+\int_{0}^{t} e^{-(t-\tau) / C}\left\|D^{k} \phi\right\|_{s-2-2 k}(\tau) d \tau\right\} .
\end{aligned}
$$

Set

$$
M_{k}=\sup _{0 \leqq \tau \leqq t}(1+\tau)^{1 / 2(k+(1 / 2))}\left\|D^{k}(U-\bar{U})\right\|_{s-2-2 k}(\tau) .
$$

First consider $k=0$, then (4.3) is simplified to

$$
\begin{aligned}
\|U-\bar{U}\|_{s-2}(t) \leqq & C\left\{(1+t)^{-1 / 4} E_{s}+\int_{0}^{t}(1+t-\tau)^{-3 / 4}\|h\|_{L^{1}}(\tau) d \tau\right. \\
& +\int_{0}^{t} e^{-(t-\tau) / C}\left\|h_{x}\right\|_{s-2}(\tau) d \tau+\int_{0}^{t}(1+t-\tau)^{-1 / 2}\left\|D^{-1} \phi\right\|(\tau) d \tau \\
& \left.+\int_{0}^{t} e^{-(t-\tau) / C}\|\phi\|_{s-2}(\tau) d \tau\right\}
\end{aligned}
$$

It is easy to see from (4.2) that

$$
\begin{aligned}
\|h\|_{L^{1}} & \leqq C\left\{\|u-\bar{u}\|^{2}(t)+\int_{0}^{t} e^{-(t-\tau) / C}\|u-\bar{u}\|^{2}(\tau) d \tau\right\} \\
& \leqq C(1+t)^{-1 / 2} M_{0}(t)^{2} .
\end{aligned}
$$

Using the Sobolev inequality and (3.5), we have

$$
\begin{aligned}
& \left\|h_{x}\right\|_{s-2}(t) \\
& \quad \leqq\left\|\left[f^{\prime}(u)-f^{\prime}(\bar{u})\right] u_{x}\right\|_{s-2}(t)+C \int_{0}^{t} e^{-(t-\tau) / C}\left\|\left[g^{\prime}(u)-g^{\prime}(\bar{u})\right] u_{x}\right\|_{s-2}(\tau) d \tau \\
& \quad \leqq C\left\{\|U-\bar{U}\|_{s}\|U-\bar{U}\|_{s-2}(t)+\int_{0}^{t} e^{-(t-\tau) / C}\|U-\bar{U}\|_{s}\|U-\bar{U}\|_{s-2}(\tau) d \tau\right\} \\
& \quad \leqq C E_{s} M_{0}(t)(1+t)^{-1 / 4} .
\end{aligned}
$$

From (4.2) we also have

$$
\begin{aligned}
\left\|D^{-1} \phi\right\|(t) & =\left\|\int_{-\infty}^{0} a^{\prime}(t-\tau)[g(\eta(\cdot, \tau))-g(\bar{u})] d \tau\right\| \\
& \leqq C E_{s} e^{-t / C}, \\
\|\phi\|_{s-2}(t) & \leqq C e^{-t / C} \sup _{\tau \leqq 0}\left\|g(\eta)_{x}\right\|_{s-2}(\tau) \leqq C E_{s} e^{-t / C} .
\end{aligned}
$$


Substitute (4.6), (4.7) and (4.8) into (4.5). We arrive at

$$
\|U-\bar{U}\|_{s-2}(t) \leqq C(1+t)^{-1 / 4}\left\{E_{s}+M_{0}(t)^{2}+E_{s} M_{0}(t)\right\} .
$$

Therefore $M_{0} \leqq C\left\{E_{s}+M_{0}^{2}(t)\right\} . M_{0}(t) \leqq C E_{s}$ if $E_{s}$ is small. Equation (1.12) is proved for $k=0$.

Now prove (1.12) by induction. Assume that (1.12) holds for $k=0,1, \ldots, k_{0}-1$. We want to prove that it holds for $k=k_{0}$. From (4.2) we have, for $k_{0} \geqq 1$,

$$
\begin{aligned}
\left\|D^{k_{0}} h\right\|_{L^{1}}(t) \leqq & \left\|D^{k_{0}-1}\left[\left(f^{\prime}(u)-f^{\prime}(\bar{u})\right) u_{x}\right]\right\|_{L^{1}}(t) \\
& +C \int_{0}^{t} e^{-(t-\tau) / C}\left\|D^{k_{0}-1}\left[\left(g^{\prime}(u)-g^{\prime}(\bar{u})\right) u_{x}\right]\right\|_{L^{1}}(\tau) d \tau .
\end{aligned}
$$

It is easy to see that the first term on the right is bounded by

$$
C\left\{\|u-\bar{u}\|\left\|D^{k_{0}} u\right\|+\sum_{j=1}^{k_{0}-1}\left\|D^{j} f^{\prime}(u)\right\|\left\|D^{k_{0}-j} u\right\|\right\} .
$$

Use induction hypothesis to bound $\left\|D^{j} f^{\prime}(u)\right\|$ and $\left\|D^{k_{0}-j} u\right\|, j=1, \ldots, k_{0}-1$. After a careful calculation we find out (4.10) is bounded by

$$
C(1+t)^{-1 / 2\left(k_{0}+1\right)}\left[E_{s} M_{k_{0}}(t)+E_{s}^{2}\right] .
$$

Similarly the second term on the right of (4.9) has the same bound. Therefore,

$$
\left\|D^{k_{0}} h\right\|_{L^{1}}(t) \leqq C(1+t)^{-1 / 2\left(k_{0}+1\right)}\left[E_{s} M_{k_{0}}(t)+E_{s}^{2}\right]
$$

Again from (4.2) we have for $k_{0} \geqq 1$,

$$
\begin{aligned}
\left\|D^{k_{0}+1} h\right\|_{s-2-2 k_{0}}(t) \leqq & \left\|D^{k_{0}}\left[\left(f^{\prime}(u)-f^{\prime}(\bar{u})\right) u_{x}\right]\right\|_{s-2-2 k_{0}}(t) \\
& +C \int_{0}^{t} e^{-(t-\tau) / C}\left\|D^{k_{0}}\left[\left(g^{\prime}(u)-g^{\prime}(\bar{u})\right) u_{x}\right]\right\|_{s-2-2 k_{0}}(\tau) d \tau .
\end{aligned}
$$

It can be estimated in a way similar to what we did for (4.9) although it is a little more complicated. For example, we have a term

$$
\begin{aligned}
\| & {\left[f^{\prime}(u)-f^{\prime}(\bar{u})\right] D^{k_{0}+1} u \|_{s-2-2 k_{0}}(t) } \\
& \leqq C\|u-\bar{u}\|^{1 / 2}\left\|u_{x}\right\|^{1 / 2}\left\|D^{k_{0}-1}(u-\bar{u})\right\|_{s-2-2\left(k_{0}-1\right)}(t) \\
& \leqq C E_{s}^{3 / 2}(1+t)^{-1 / 2\left(k_{0}-1 / 4\right)}\left\|u_{x}\right\|^{1 / 2} \\
& \leqq C E_{s}^{3 / 2}(1+t)^{-1 / 2\left(k_{0}+(1 / 2)\right)}\left[M_{k_{0}}(t)^{1 / 2}+E_{s}^{1 / 2}\right]
\end{aligned}
$$

Here in the last step we have considered $k_{0}=1$ and $k_{0}>1$. After a careful computation we get

$$
\left\|D^{k_{0}+1} h\right\|_{s-2-2 k_{0}}(t) \leqq C(1+t)^{-1 / 2\left(k_{0}+(1 / 2)\right)}\left[E_{s}^{2}+E_{s} M_{k_{0}}(t)\right] .
$$

Substitute (4.6), (4.11), (4.12) and (4.8) into (4.3). We have

$$
\left\|D^{k_{0}}(U-\bar{U})\right\|_{s-2-2 k_{\dot{0}}}(t) \leqq C(1+t)^{-1 / 2\left(k_{0}+(1 / 2)\right)}\left\{E_{s}+E_{s} M_{k_{0}}(t)+E_{s}^{2}\right\} .
$$

Therefore,

$$
M_{k_{0}}(t) \leqq C\left\{E_{s}+E_{s} M_{k_{0}}(t)+E_{s}^{2}\right\}
$$


We conclude that $M_{k_{0}}(t) \leqq C E_{s}$ if $E_{s}$ is small. Equation (1.12) is proved. Q.E.D Proof of Theorem 1.4. Linearize both (1.1) and (1.13) around the constant state $\bar{U}$. We have (4.1), (4.2) and

$$
\left\{\begin{array}{l}
(\tilde{u}-\bar{u})_{t}-(\tilde{v}-\bar{v})_{x}=\frac{\mu}{2}(\tilde{u}-\bar{u})_{x x} \\
(\tilde{v}-\bar{v})_{t}-p^{\prime}(\bar{u})(\tilde{u}-\bar{u})_{x}=\frac{\mu}{2}(\tilde{v}-\bar{v})_{x x}+\tilde{h}_{x}
\end{array}\right.
$$

where

$$
\tilde{h}=p(\tilde{u})-p(\bar{u})-p^{\prime}(\bar{u})(\tilde{u}-\bar{u})
$$

From Theorem 2.6 we have, for $0 \leqq k \leqq j \leqq s-4-k$,

$$
\begin{aligned}
\left\|D^{j}(U-\tilde{U})\right\|(t) \leqq & C\left[(1+t)^{-1 / 2(j+(3 / 2))}\left\|U_{0}-\bar{U}\right\|_{L^{1}}+e^{-t / C}\left\|U_{0}-\bar{U}\right\|_{j+1}\right. \\
& +\int_{0}^{t / 2}(1+t-\tau)^{-1 / 2(j+(3 / 2))}\|h-\tilde{h}\|_{L^{1}}(\tau) d \tau \\
& +\int_{t / 2}^{t}(1+t-\tau)^{-1 / 2(j-k+(3 / 2))}\left\|D^{k}(h-\tilde{h})\right\|_{L^{1}}(\tau) d \tau \\
& +\int_{0}^{t}(1+t-\tau)^{-1 / 2(j+(1 / r)+(1 / 2))}\left\|D^{-1} \phi\right\|_{L^{r}}(\tau) d \tau \\
& +\int_{0}^{t / 2}(1+t-\tau)^{-1 / 2(j+(5 / 2))}\|\tilde{h}\|_{L^{1}}(\tau) d \tau \\
& +\int_{t / 2}^{t}(1+t-\tau)^{-1 / 2(j-k+(3 / 2))}\left\|D^{k+1} \tilde{h}\right\|_{L^{1}}(\tau) d \tau \\
& \left.+\int_{0}^{t} e^{-(t-\tau) / C}\left(\left\|D^{j} \phi\right\|+\left\|D^{j+1} h\right\|+\left\|D^{j+1} \tilde{h}\right\|_{1}\right)(\tau) d \tau\right] .
\end{aligned}
$$

$\operatorname{Sum} \operatorname{up} j$

$$
\begin{aligned}
\left\|D^{k}(U-\tilde{U})\right\|_{s-4-2 k}(t) \leqq & C\left\{(1+t)^{-1 / 2(k+(3 / 2))} E_{s}\right. \\
& +\int_{0}^{t / 2}(1+t-\tau)^{-1 / 2(k+(3 / 2))}\|h-\tilde{h}\|_{L^{1}}(\tau) d \tau \\
& +\int_{t / 2}^{t}(1+t-\tau)^{-3 / 4}\left\|D^{k}(h-\tilde{h})\right\|_{L^{1}}(\tau) d \tau \\
& +\int_{0}^{t / 2}(1+t-\tau)^{-1 / 2(k+(5 / 2))}\|\tilde{h}\|_{L^{1}}(\tau) d \tau \\
& +\int_{t / 2}^{t}(1+t-\tau)^{-3 / 4}\left\|D^{k+1} \tilde{h}\right\|_{L^{1}}(\tau) d \tau
\end{aligned}
$$




$$
\begin{aligned}
& +\int_{0}^{t}(1+t-\tau)^{-1 / 2(k+(1 / r)+(1 / 2))}\left\|D^{-1} \phi\right\|_{L^{r}}(\tau) d \tau \\
& +\int_{0}^{t} e^{-(t-\tau) / C}\left[\|\phi\|_{s-4-k}+\left\|D^{k+1} h\right\|_{s-4-2 k}\right. \\
& \left.\left.+\left\|D^{k+1} \tilde{h}\right\|_{s-3-2 k}\right](\tau) d \tau\right\}
\end{aligned}
$$

where $h$ and $\phi$ are given by (4.2). Set

$$
M_{k}(t)=\sup _{0 \leqq \tau \leqq t}(1+\tau)^{1 / 2(k+(1 / r)+(1 / 2))}\left\|D^{k}(U-\tilde{U})\right\|_{s-4-2 k}(\tau) .
$$

First consider $k=0$. Equation (4.15) becomes

$$
\begin{aligned}
\|U-\tilde{U}\|_{s-4}(t) \leqq & C\left\{(1+t)^{-3 / 4} E_{s}+\int_{0}^{t}(1+t-\tau)^{-3 / 4}\|h-\tilde{h}\|_{L^{1}}(\tau) d \tau\right. \\
& +\int_{0}^{t / 2}(1+t-\tau)^{-5 / 4}\|\tilde{h}\|_{L^{1}}(\tau) d \tau+\int_{t / 2}^{t}(1+t-\tau)^{-3 / 4}\left\|\tilde{h}_{x}\right\|_{L^{1}}(\tau) d \tau \\
& +\int_{0}^{t}(1+t-\tau)^{-1 / 2((1 / r)+(1 / 2))}\left\|D^{-1} \phi\right\|_{L^{r}}(\tau) d \tau \\
& \left.+\int_{0}^{t} e^{-(t-\tau) / C}\left[\|\phi\|_{s-4}+\left\|h_{x}\right\|_{s-4}+\left\|\tilde{h}_{x}\right\|_{s-3}\right](\tau) d \tau\right\}
\end{aligned}
$$

Integrating by part for $h$, we have

$$
\begin{aligned}
h-\tilde{h}= & p(u)-p(\tilde{u})-p^{\prime}(\bar{u})(u-\tilde{u})+a(t)\left[g\left(u_{0}\right)-g(\bar{u})-g^{\prime}(\bar{u})\left(u_{0}-\bar{u}\right)\right] \\
& +\int_{0}^{t} a(t-\tau)\left[g^{\prime}(u)-g^{\prime}(\bar{u})\right] v_{x}(x, \tau) d \tau .
\end{aligned}
$$

It is easy to see that

$$
\left|p(u)-p(\tilde{u})-p^{\prime}(\bar{u})(u-\tilde{u})\right| \leqq C(|u-\bar{u}|+|\tilde{u}-\bar{u}|)|u-\tilde{u}| .
$$

Therefore by Theorem 1.1 and Theorem 1.3i), we have

$$
\|h-\tilde{h}\|_{L^{1}}(t) \leqq C\left\{E_{s}(1+t)^{-1 / 4}\|u-\tilde{u}\|+e^{-t / C} E_{s}^{2}+E_{s}^{2}(1+t)^{-1}\right\} .
$$

Similarly we also have

$$
\begin{aligned}
& \|\tilde{h}\|_{L^{1}}(t) \leqq C E_{s}^{2}(1+t)^{-1 / 2} \\
& \left\|\tilde{h}_{x}\right\|_{L^{1}}(t),\left\|h_{x}\right\|_{s-4}(t),\left\|\tilde{h}_{\|^{\prime}}\right\|_{s-3}(t) \leqq C E_{s}^{2}(1+t)^{-1}, \\
& \left\|D^{-1} \phi\right\|_{L^{r}}(t) \leqq C e^{-t / C} E_{s, r}
\end{aligned}
$$

Substitute (4.19), (4.20) and (4.8) into (4.17),

$$
\|U-\tilde{U}\|_{s-4}(t) \leqq C(1+t)^{-1 / 2((1 / r)+(1 / 2))}\left\{E_{s, r}+E_{s, r} M_{0}(t)\right\} .
$$

Therefore,

$$
M_{0}(t) \leqq C\left\{E_{s, r}+E_{s, r} M_{0}(t)\right\}
$$


We conclude that $M_{0}(t) \leqq C E_{s, r}$ if $E_{s, r}$ is small. Equation (1.23) is proved for $k=0$.

Prove (1.23) by induction for $k \geqq 1$. Suppose (1.23) holds for $k=0,1, \ldots, k_{0}-1$, where $k_{0} \geqq 1$. Then by (4.18),

$$
\begin{aligned}
\left\|D^{k_{0}}(h-\tilde{h})\right\|_{L^{1}}(t) \leqq & \left\|p^{\prime}(u) D^{k_{0}} u-p^{\prime}(\tilde{u}) D^{k_{0}} \tilde{u}-p^{\prime}(\bar{u})\left[D^{k_{0}} u-D^{k_{0}} \tilde{u}\right]\right\|_{L^{1}}(t) \\
& +C \sum_{l=1}^{k_{0}-1}\left\|D^{l} p^{\prime}(u) D^{k_{0}-l} u-D^{l} p^{\prime}(\tilde{u}) D^{K_{0}-l} \tilde{u}\right\|_{L^{1}} \\
& +C e^{-t / C}\left\|D^{k_{0}} g\left(u_{0}\right)-g^{\prime}(\bar{u}) D^{k_{0}} u_{0}\right\|_{L^{1}} \\
& +C \int_{0}^{t} e^{-(t-\tau) / C}\left\|D^{k_{0}}\left\{\left[g^{\prime}(u)-g^{\prime}(\bar{u})\right] v_{x}\right\}\right\|_{L^{1}}(\tau) d \tau .
\end{aligned}
$$

Take Taylor expansion of $p^{\prime}(u)$ around $\tilde{u}$. We see that the first term is bounded by $\left\|\left(p^{\prime}(\tilde{u})-p^{\prime}(\bar{u})\right)\left(D^{k_{0}} u-D^{k_{0}} \tilde{u}\right)\right\|_{L^{1}}+C\left\|(u-\tilde{u}) D^{k_{0}} u\right\|_{L^{1}}$, hence by $C E_{s} M_{k_{0}}(t)(1+t)^{-1 / 2\left(k_{0}+(1 / r)+1\right)}+C E_{s, r}(1+t)^{-1 / 2\left(k_{0}+(1 / r)+1\right)}$. Here we have used Theorem 1.3 i), (4.16), (1.23) with $k=0$ and Theorem 1.1. Similarly using the induction hypothesis we can bound the other terms by $C E_{s, r}(1+t)^{-1 / 2\left(k_{0}+(1 / r)+1\right)}$. Therefore

$$
\left\|D^{k_{0}}(h-\tilde{h})\right\|_{L^{1}}(t) \leqq C\left\{E_{s} M_{k_{0}}(t)(1+t)^{-1 / 2\left(k_{0}+(1 / r)+1\right)}+E_{s, r}(1+t)^{-1 / 2\left(k_{0}+(1 / r)+1\right)}\right\} .
$$

By Theorem 1.1 and Theorem 1.3 i) we also have

$$
\left\|D^{k_{0}+1} \tilde{h}\right\|_{L^{1}}(t), \quad\left\|D^{k_{0}+1} h\right\|_{s-4-2 k_{0}}(t), \quad\left\|D^{k_{0}+1} \tilde{h}\right\|_{s-3-2 k_{0}}(t) \leqq C E_{s}(1+t)^{-1 / 2\left(k_{0}+2\right)} \text {. }
$$

Substitute (4.19), (4.21), (4.20), (4.22) and (4.8) into (4.15),

$$
\left\|D^{k_{0}}(U-\tilde{U})\right\|_{s-4-2 k_{0}}(t) \leqq C(1+t)^{-1 / 2\left(k_{0}+(1 / r)+(1 / 2)\right)}\left\{E_{s, r}+E_{s} M_{k_{0}}(t)\right\} .
$$

Therefore

$$
M_{k_{0}}(t) \leqq C\left\{E_{s, r}+E_{s, r} M_{k_{0}}(t)\right\} .
$$

$M_{k_{0}}(t) \leqq C E_{s, r}$ if $E_{s, r}$ is small. Equation (1.23) is proved for $k=k_{0}$. Q.E.D.

Acknowledgements. This work represents a portion of my thesis. I would like to thank my advisor. Prof. Tai-Ping Liu for his help and guidance during the preparation of the thesis. I would also like to thank Prof. John A. Nohel for his interest in this work and for his suggestions on improving the wording of this paper. The research was supported in part by the AFOSR under Grant No. 89-0203.

\section{References}

1. Liu, T.-P.: Nonlinear Waves for Viscoelasticity with Fading Memory. J. Differ. Eqs. 76, 26-46 (1988)

2. Kawashima, S.: Large-time Behavior of Solutions to Hyperbolic-Parabolic Systems of Conservation Laws and Applications. Proc. Roy. Soc. Edinburgh 106 A, 169-194 (1987)

3. Liu, T.-P.: Interactions of Nonlinear Hyperbolic Waves. In: Liu, F.-C., Liu, T.-P. (eds.) Nonlinear Analysis, pp. 171-184. Singapore: World Scientific 1991

4. Chern, I.-L., Liu, T.-P.: Convergence to Diffusion Waves of Solutions for Viscous Conservation Laws. Commun. Math. Phys. 110, 503-517 (1987), 120, 525-527 (1989) 
5. Hrusa, W., Nohel, J.: The Cauchy Problem in One-Dimensional Nonlinear Viscoelasticity. J. Differ. Eqs. 59, 388-412 (1985)

6. Liu, T.-P.: Nonlinear Stability of Shock Waves for Viscous Conservation Laws. Mem. Am. Math. Soc. 328, 1-108 (1985)

7. Renardy, M., Hrusa, W., Nohel, J. A.: Mathematical Problems in Viscoelasticity. England: Longman Sci. and Tech. New York: Wiley 1987

8. Dafermos, C. M., Nohel, J. A.: A Nonlinear Hyperbolic volterra Equation in Viscoelasticity. In: Clark, D. N., Pecelli, G., Sacksteder, R. (eds.) Contributions to Analysis and Geometry. Am. J. Math. [Suppl] pp. 87-116. Baltimore, London: The Johns Hopkins University Press 1981

Communicated by A. Jaffe 
